\title{
OBSCURATION IN EXTREMELY LUMINOUS QUASARS
}

\author{
M. Polletta, ${ }^{1,2}$ D. Weedman, ${ }^{3}$ S. Hönig, ${ }^{4}$ C. J. Lonsdale, ${ }^{1,5}$ H. E. Smith, ${ }^{1,6}$ and J. HoucK ${ }^{3}$ \\ Received 2007 April 11; accepted 2007 September 26
}

\begin{abstract}
The SEDs and IR spectra of a remarkable sample of obscured AGNs selected in the MIR are modeled with recent clumpy torus models. The sample contains 21 AGNs at $z=1.3-3$ discovered in the largest Spitzer surveys (SWIRE, NDWFS, and FLS) by means of their extremely red IR to optical colors. All sources show the $9.7 \mu \mathrm{m}$ silicate feature in absorption and have extreme MIR luminosities $\left[L(6 \mu \mathrm{m}) \simeq 10^{46} \mathrm{ergs} \mathrm{s}^{-1}\right]$. The IR SEDs and spectra of 12 sources are well reproduced with a simple torus model, while the remaining nine sources require foreground extinction from a cold dust component to reproduce both the depth of the silicate feature and the NIR emission from hot dust. The best-fit torus models show a broad range of inclinations. Based on the unobscured QSO MIR luminosity function (Brown and coworkers) and on a color-selected sample of AGNs, we estimate the surface densities of obscured and unobscured QSOs with $L(6 \mu \mathrm{m})>10^{12} L_{\odot}$ and $z=1.3-3.0$ to be about $17-22$ and $11.7 \mathrm{deg}^{-2}$, respectively. Overall we find that $\sim 35 \%-41 \%$ of luminous QSOs are unobscured, $37 \%-40 \%$ are obscured by the torus, and $23 \%-25 \%$ are obscured by a cold absorber detached from the torus. These fractions are consistent with a decrease of the torus covering fraction at large luminosities as predicted by receding torus models. An FIR component is observed in eight objects with luminosity greater than $3.3 \times 10^{12} L_{\odot}$, implying SFRs of $600-3000 M_{\odot} \mathrm{yr}^{-1}$. In the whole sample, the average contribution from a starburst component to the bolometric luminosity, as estimated from the PAH $7.7 \mu \mathrm{m}$ luminosity in the composite IR spectra, is $\leq 20 \%$ of the total bolometric luminosity.
\end{abstract}

Subject headings: galaxies: active — galaxies: high-redshift — infrared: galaxies — quasars: general

Online material: color figures

\section{INTRODUCTION}

\subsection{Obscured AGNs at High Luminosities}

Multiple X-ray studies show that the fraction of active galactic nuclei (AGNs) whose emission is heavily absorbed $\left(N_{\mathrm{H}} \geq\right.$ $10^{22} \mathrm{~cm}^{-2}$ ) decreases with increasing luminosity (from $>80 \%$ at $L_{\mathrm{X}}=10^{42} \mathrm{ergs} \mathrm{s}^{-1}$ to $38 \%$ at $10^{45} \mathrm{ergs} \mathrm{s}^{-1}$; Akylas et al. 2006). However, because of the difficulty in detecting and identifying absorbed AGNs, it is still unclear whether their paucity at high luminosities is an observational selection effect or real (La Franca et al. 2005; Treister \& Urry 2006; Akylas et al. 2006; Tozzi et al. 2006). In order to overcome these uncertainties, searches for absorbed QSOs have focused on observations at wavelengths less affected by absorption, i.e., infrared (IR) and radio, e.g., FIRST, 2MASS, and various Spitzer surveys (e.g., Polletta et al. 2006; Martínez-Sansigre et al. 2006; Lacy et al. 2007; Wilkes et al. 2002; Urrutia et al. 2005).

These searches have unveiled a large population of QSOs obscured at optical wavelengths. Assuming that all optically obscured QSOs are also absorbed in X-rays, the fraction of absorbed QSOs would be $\geq 50 \%$ of all QSOs (Martínez-Sansigre et al. 2005). This fraction is still significantly lower than the fraction measured for AGNs at lower luminosities, 80\% (Osterbrock \& Martel 1993; Akylas et al. 2006).

Current AGN evolutionary models and observations indicate a link between the absorption properties in AGNs and their lu-

\footnotetext{
1 Center for Astrophysics and Space Sciences, University of California, San Diego, La Jolla, CA 92093-0424.

2 Institut d'Astrophysique de Paris, 75014 Paris, France; polletta@iap.fr.

3 Department of Astronomy, Cornell University, Ithaca, NY 14853.

4 Max-Planck-Institut für Radioastronomie, 53121 Bonn, Germany.

5 Infrared Processing and Analysis Center, California Institute of Technology, Pasadena, CA 91125.

6 Deceased 2007 August 16.
}

minosities (see, e.g., Fig. 4 in Hopkins et al. 2005). More specifically, AGNs in a growing phase are moderately luminous and heavily absorbed, and as the AGN luminosity increases, the intense radiation field destroys the surrounding matter and the AGN shines unabsorbed (e.g., Di Matteo et al. 2005). A similar trend is predicted by the receding torus models (e.g., Lawrence 1991; Simpson 2005). According to these models, the opening angle of the torus (measured from the torus axis to the equatorial plane) is larger in more luminous objects. Thus, obscuration should be less common in more luminous AGNs.

\subsection{The Obscuring Matter in AGNs}

Obscuration in AGNs is caused by a mixture of neutral and ionized gas, as well as dust. Absorption by gas is usually observed in the X-rays through the suppression of soft X-ray emission with an energy cutoff that increases with the gas column density, $N_{\mathrm{H}}$. The absorbing gas is mainly located in the circumnuclear region as suggested by measured variability (Risaliti et al. 2005, 2007), but gas in our Galaxy and in the host galaxy can also contribute to the overall absorption. Obscuration by dust is usually observed at optical and IR wavelengths where the continuum and broad emission lines are reddened and, in some cases, completely suppressed. In the near- and mid-IR (NIR and MIR), dust obscuration interplays with thermal reemission from the putative parsec-scaled dust torus. Depending on the actual geometry, optical depth, and chemistry, dust absorption features at about 10 and $18 \mu \mathrm{m}$ due to silicates (Si) can be present in the IR.

According to AGN unification models (e.g., Antonucci 1993) and torus models (e.g., Krolik \& Begelman 1988; Pier \& Krolik 1992; Efstathiou \& Rowan-Robinson 1995; Granato et al. 1997; Nenkova et al. 2002; Dullemond \& van Bemmel 2005; Fritz et al. 2006; Hönig et al. 2006), optical depths are expected to be at least roughly correlated from the X-ray through the MIR, although this correlation might be very weak for clumpy tori. In obscured 
AGNs the line of sight (LOS) intercepts the putative torus and thus absorbs radiation from the nucleus (broad emission lines, $\mathrm{X}$-rays, and thermal radiation from hot dust), while in unobscured AGNs the radiation from the nucleus is directly visible and thus unabsorbed. An edge-on view of the torus is characterized by red optical through IR colors and Si features in absorption (at 10 and $18 \mu \mathrm{m}$ ), while a face-on torus has bluer colors and Si features absent or in emission. Sources with narrow emission lines in their optical spectra, red colors, and strong Si absorption features are usually associated with a highly inclined torus and thus are expected to be also absorbed in the X-rays. However, there is emerging evidence for a surprising mismatch between the absorption measured in the X-rays and that measured in the optical through IR for a significant fraction of sources, especially at high luminosities.

AGNs selected in the MIR because of extremely red optical through IR colors sometimes show broad optical emission lines, even having absorption features in their IR spectra in some cases (Brand et al. 2007). The comparison between X-ray absorption and optical obscuration in various X-ray-selected AGN samples shows that about $20 \%-30 \%$ of obscured AGNs are not absorbed in the X-rays and vice versa (e.g., Perola et al. 2004; Tozzi et al. 2006; Tajer et al. 2007; Gliozzi et al. 2007). The MIR spectra of a sample of type 2 QSOs with heavy X-ray absorption did not reveal the Si feature in absorption at $10 \mu \mathrm{m}$ (Sturm et al. 2006) as expected. This mismatch is even more pronounced at high luminosities. Indeed, absorption signatures in the IR, e.g., the $\mathrm{Si}$ absorption feature at $10 \mu \mathrm{m}$, are more prominent at the high luminosities of ultraluminous infrared galaxies (ULIRGS; Sanders \& Mirabel 1996; Hao et al. 2007), while X-ray absorption is progressively less common in AGNs with increasing luminosity (Ueda et al. 2003; Hasinger et al. 2005). These results suggest that a nonnegligible fraction of obscured AGNs might not be obscured by a torus, but by dust in either the narrow-line region (NLR; Sturm et al. 2005) or the host galaxy, and that large X-ray column densities are not always associated with geometrically and optically thick dust distributions (see also Rigby et al. 2006; Martínez-Sansigre et al. 2006). Alternatively, this mismatch can be explained by a low dust-to-gas ratio $\left(A_{V} / N_{\mathrm{H}}\right)$ or by a different path for the IR and the X-ray LOSs (Maiolino et al. 2001; Shi et al. 2006).

Large optical depths for Si absorption features $(>1.7)$ usually imply a compact source deeply embedded in a smooth distribution of material that is both geometrically and optically thick, rather than absorption by a foreground screen of dust close to the heating source (Levenson et al. 2007; Imanishi et al. 2007). Also a detached foreground cold absorber that is far enough from the heating source to be cold can produce large Si optical depths. $\operatorname{Deep}\left(\tau_{\mathrm{Si}}>1.7\right) \mathrm{Si}$ absorption features are usually considered as indicators of a buried compact source such as an AGN. However, it is not clear whether the optical depth is a consequence of a random alignment of Si clouds or a specific dust distribution and orientation. The observed MIR spectra of obscured AGNs do not favor the latter scenario because the Si absorption features are not always present in the spectra of optically obscured and X-ray absorbed AGNs, as would be expected if the Si absorbers were associated with the same material that suppresses the broad emission lines and the soft X-ray emission.

In order to investigate the properties of the obscuring matter in extremely luminous AGNs and asses how often the absorption signatures at optical, X-ray, and infrared wavelengths do not correlate, a comprehensive analysis of the absorption properties at all wavelengths of obscured AGNs at high luminosity is necessary, as well as detailed studies of their absorbing matter. Observations at various wavelengths from X-ray through optical and IR constrain the absorption along multiple LOSs and thus the geometry and distribution of the absorbing matter.

In this work, we investigate the observed properties of the obscuring matter in a sample of obscured and extremely luminous AGNs, and we model their spectral energy distributions (SEDs) with clumpy torus models (Hönig et al. 2006) to explore the dust geometries associated with large obscuration. This study is based on a sample of extremely luminous and obscured AGNs for which multiwavelength SEDs and IR spectra are available. The selected sample includes sources from the three widest Spitzer extragalactic surveys that were observed with the Spitzer Infrared Spectrograph (IRS; Houck et al. 2004). In $\S 2$ we describe the sample selection, and in $\S 3$ we present the observations and the data used in this work. The IRS spectra and detected spectral features are presented in $\S 4$. The modeling of the SEDs using clumpy torus models (Hönig et al. 2006) is presented in $\S 5$. The composite spectra of two subsamples defined on the basis of the model results are analyzed in $\S 6$. The properties (IR colors, surface density, luminosity, optical depths, and redshift distribution) of the selected sample are compared with those of other samples of AGNs and ULIRGs from the literature in $\S 7$. The X-ray properties and a comparison between the absorption seen in the X-ray and the optical depth in the IR are described in $\S 8$. Our results and their implications are discussed in $\S 9$ and summarized in $\S 10$.

Throughout the paper, the term "absorbed" refers to X-ray sources with effective column densities $N_{\mathrm{H}} \geq 10^{22} \mathrm{~cm}^{-2}$, and "obscured" to sources with red optical-IR colors, e.g., $F(3.6 \mu \mathrm{m}) /$ $F\left(r^{\prime}\right)>20$ or $F(24 \mu \mathrm{m}) / F\left(r^{\prime}\right)>100$, implying extreme dust extinction. The IR SEDs are defined as "red" if they are as red or redder than a power-law model, $F_{\nu} \propto \nu^{-\alpha}$, with slope $\alpha=2$. The terms type 1 and type 2 refer to AGNs with broad and narrow optical emission lines, respectively, in their optical spectra. We adopt a flat cosmology with $H_{0}=71 \mathrm{~km} \mathrm{~s}^{-1} \mathrm{Mpc}^{-1}, \Omega_{M}=$ 0.27 , and $\Omega_{\Lambda}=0.73$ (Spergel et al. 2003).

\section{SAMPLE DESCRIPTION}

There are three large-area Spitzer surveys with the Infrared Array Camera (IRAC; Fazio et al. 2004), the Multiband Imaging Photometer (MIPS; Rieke et al. 2004), and optical photometric coverage, the $50 \mathrm{deg}^{2}$ Spitzer Wide-Area Infrared Extragalactic Survey ${ }^{7}$ (SWIRE; Lonsdale et al. 2003, 2004); the $9 \mathrm{deg}^{2}$ NOAO Deep Wide-Field Survey (NDWFS) of the Bootes field (Jannuzi \& Dey 1999; Murray et al. 2005); and the $3.7 \mathrm{deg}^{2}$ Extragalactic First Look Survey ${ }^{8}$ (E-FLS; Fadda et al. 2006).

The initial objective for Spitzer IRS spectroscopy of sources chosen from these surveys was to understand populations of new sources that would not have been known prior to Spitzer. Consequently, spectroscopy has emphasized sources that are optically very faint, typically $\operatorname{mag}(R)>24$, and IR-bright, i.e., $F(24 \mu \mathrm{m})>$ 1 mJy. Various selection criteria based on IRAC colors also entered choices of spectroscopic targets, but the unifying theme of existing spectroscopic samples is their high ratio of MIR to optical flux, IR/opt $=\nu F_{\nu}(24 \mu \mathrm{m}) / \nu F_{\nu}(R)$. Most sources chosen for spectroscopy have IR/opt $\geq 10$. For 58 sources observed in Bootes (Houck et al. 2005; Weedman et al. 2006c), 70 sources observed in the FLS (Yan et al. 2007; Weedman et al. 2006b), and 20 sources in the SWIRE Lockman Hole field (Weedman et al. 2006a), the majority are at high redshift $(z \sim 2)$ and many have infrared spectra dominated by the strong silicate ( $\mathrm{Si}$ ) absorption feature at rest frame $9.7 \mu \mathrm{m}$. The presence of this absorption combined with

\footnotetext{
7 See http://swire.ipac.caltech.edu/swire/swire.html.

8 See http://ssc.spitzer.caltech.edu/fls/.
} 
the red colors and high luminosities of these sources led these previous observers to the conclusion that IR-bright sources with large IR/optical flux ratios are dominated by obscured AGNs (or QSOs because of their very high luminosities). A minority of sources in these studies show strong PAH emission features characteristic of starbursts, but these sources generally do not show strong Si absorption, so different populations of AGNs and starbursts are observed.

If the interpretation of the obscured sources as AGNs is correct, this implies a significant population of luminous, obscured QSOs. It is essential, therefore, to verify the AGN classification and to understand the obscured sources in the context of models for AGNs.

Our goal in this work is to determine quantitative AGN models for the most luminous of these obscured sources, with two primary objectives. The first is to investigate the properties of the AGN emission and the nature of the obscuration in AGNs at high luminosities. The second is to use the SEDs to define the color criteria that describe such obscured AGNs in the IRAC and MIPS color space in order to compare surface densities between obscured and unobscured QSOs at high luminosities and high redshifts. To achieve these goals, we have selected for detailed modeling the most luminous of the obscured sources with AGN-dominated MIR SEDs from the archival Spitzer spectroscopy in the survey fields mentioned.

Our selection criteria are the following: high redshift, i.e., $z>1$; large MIR luminosities, i.e., $6 \mu \mathrm{m}$ luminosities, $\nu L_{\nu}(6 \mu \mathrm{m})=$ $L(6 \mu \mathrm{m}) \geq 10^{12} L_{\odot}$; the presence of $\mathrm{Si}$ in absorption in the IRS spectra; and red MIR SEDs consistent with being AGN dominated (see, e.g., Polletta et al. 2006; Alonso-Herrero et al. 2006). There are 21 sources that satisfy all these criteria; 13 are from the literature (Houck et al. 2005; Weedman et al. 2006a; Yan et al. 2007), and 8 are from our own IRS observing programs. As a consequence of these selection criteria, all sources have $24 \mu \mathrm{m}$ fluxes greater than $1 \mathrm{mJy}$ and have relatively large IR/optical flux ratios, i.e., $F(24 \mu \mathrm{m}) / F\left(r^{\prime}\right) \geq 400$. To our knowledge, this sample contains all sources with available IRS spectra in the literature or the Spitzer public archive that satisfy the selection criteria described. However, this sample is not meant to be complete or unbiased since it is mainly based on the availability of IRS spectra, therefore it is not essential to be exhaustive.

The selected sample includes the most luminous obscured QSOs currently known. The large MIR luminosities of the selected targets enable us to measure the emission from the hottest dust in the torus with negligible contribution from the host galaxy, providing a laboratory to test the predictions of torus models in obscured AGNs. Up to now, these kinds of SEDs could be sampled only using high spatial resolution data in nearby AGNs (e.g., in NGC 1068; Hönig et al. 2006). By combining samples from the widest Spitzer surveys, we were able to find a significant number of these rare objects.

The list of selected sources, coordinates, and optical and IR fluxes are reported in Table 1. For simplicity, we use throughout the paper simplified names for the selected sources. Official IAU names are reported in Table 1. For the SWIRE sources, the first two letters in their names designate the field where they were discovered (LH for Lockman Hole, N1 for ELAIS-N1, and N2 for ELAIS-N2). The first letter of the NDWFS source names is B for Bootes. The names of the FLS sources correspond to those in Yan et al. (2007).

\section{OBSERVATIONS AND DATA ANALYSIS}

Multiband photometric data in the optical from the ground and in the IR from Spitzer are available for all sources. The available data and references are listed in Table 1. All sources are detected with MIPS at $24 \mu \mathrm{m}$ with fluxes ranging from 1.0 to $10.6 \mathrm{mJy}$ and a median flux of $2.6 \mathrm{mJy}$. All sources have been observed with IRAC in four bands, from 3.6 to $8.0 \mu \mathrm{m}$ with varying limits. The FLS sources have few detections with IRAC, probably because of their less sensitive observations compared to the NDWFS and SWIRE surveys. Eight sources have been observed in five optical bands, from $U$ to $z$ band, three in four bands, five in three bands, and five in one band. Optical magnitudes for Bootes sources are in NDWFS Data Release 3 (DR3). Optical and IR fluxes for the FLS sources were obtained from Yan et al. (2007) and Sajina et al. (2007a), and those for the SWIRE sources were taken from the latest internal catalogs that will be released in the Data Release 6 (for details on the data reduction see Surace et al. 2005). ${ }^{9}$

The IRAC fluxes of the sources in the NDWFS were measured from the post-BCD images in the Spitzer archive. Aperture fluxes were measured within a $2.9^{\prime \prime}$ radius aperture at their position and background subtracted. Aperture corrections as derived from the SWIRE survey were applied. The aperture corrections are 1.15, $1.15,1.25$, and 1.43 for the four IRAC bands, respectively.

All sources have been observed in the optical $r^{\prime}$ or $R$ band, and $16 / 21$ have been detected. The $F(24 \mu \mathrm{m}) / F(R)$ flux ratio ranges from 575 to 25,000 with a median value of 3500 .

IRS data were taken in part from the archive and in part from our own projects (Houck et al. 2005; Weedman et al. 2006a). The spectroscopic observations were made with the IRS Short Low module in order 1 (SL1) and with the Long Low module in orders 1 and 2 (LL1 and LL2), described in Houck et al. (2004), ${ }^{10}$ providing low-resolution spectral coverage from $\sim 8$ to $\sim 35 \mu \mathrm{m}$. The main parameters of the IRS observations are listed in Table 2.

Since the objects have similar properties in terms of SED and fluxes, we applied the same reduction procedure to all spectra for uniformity. The reduction method is described in detail in Weedman et al. (2006a) and briefly summarized here. Six SWIRE sources (see details in Table 2) were processed with version 11.0 of the SSC pipeline; the remaining sources were processed with version 13.0. Extraction of source spectra was done with the SMART analysis package (Higdon et al. 2004). Since the selected sources are faint and the spectra are dominated by background signal, we restricted the number of pixels used to define the source spectrum to a width of only 4 pixels (scaling with wavelength) in order to improve the spectral signal-to-noise ratio $(\mathrm{S} / \mathrm{N})$. The flux correction necessary to change the fluxes obtained with the narrow extraction to the fluxes that would be measured with the standard extraction provided for the basic calibrated data was measured by extracting an unresolved source of high $\mathrm{S} / \mathrm{N}$ with both techniques, and this correction (typically $\sim 10 \%$ but varying with wavelength) was applied to all sources.

A search in the literature provided additional data for six sources in the sample, an Infrared Space Observatory (ISO) $15 \mu \mathrm{m}$ flux measurement for N2_08 (González-Solares et al. 2005), and MAMBO $1.2 \mathrm{~mm}$ flux upper limits for all five E-FLS sources (Lutz et al. 2005).

The SEDs and IRS spectra of all sources in the sample are shown in Figures 1 and 2. The displayed spectra have been boxcar smoothed to 9 times the approximate resolution of the different IRS modules $(0.6 \mu \mathrm{m}$ for SL1, $0.9 \mu \mathrm{m}$ for LL2, and $1.2 \mu \mathrm{m}$ for

\footnotetext{
9 Available at http://data.spitzer.caltech.edu/popular/swire/20050603_ enhanced_v1/Documents/SWIRE2_doc_083105.pdf.

${ }^{10}$ The IRS was a collaborative venture between Cornell University and Ball Aerospace Corporation funded by NASA through the Jet Propulsion Laboratory and the Ames Research Center.
} 


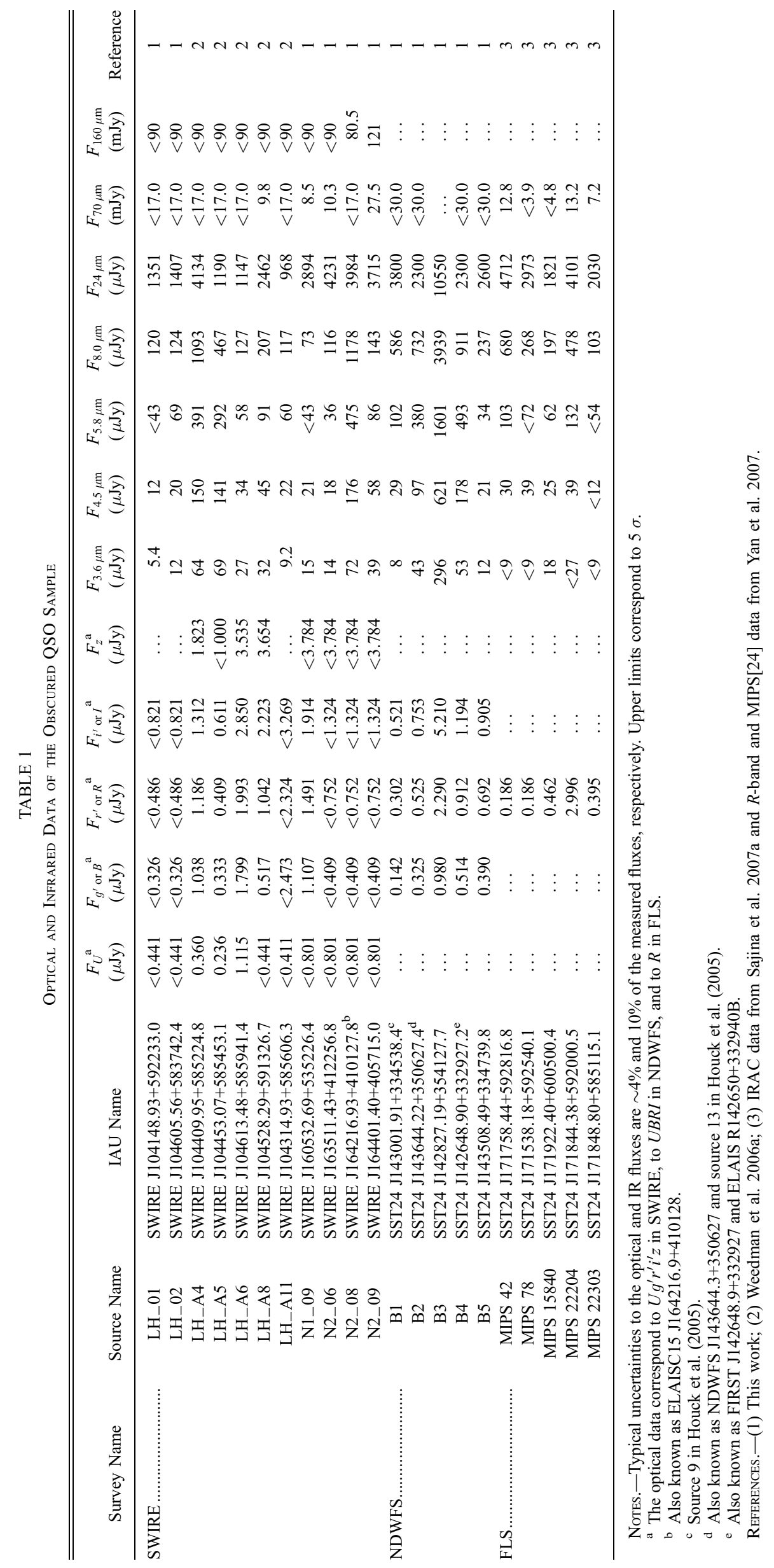


TABLE 2

IRS Low-Resolution Spectroscopy Observation Log

\begin{tabular}{|c|c|c|c|c|}
\hline Source Name & Program ID & $\begin{array}{c}\text { SL1 } \\
\text { (s) }\end{array}$ & $\begin{array}{c}\text { LL2 } \\
\text { (s) }\end{array}$ & $\begin{array}{c}\text { LL1 } \\
\text { (s) }\end{array}$ \\
\hline LH_01 .. & 136 & $3 \times 60$ & $6 \times 120$ & $6 \times 120$ \\
\hline LH_02 ... & 136 & $3 \times 60$ & $6 \times 120$ & $6 \times 120$ \\
\hline $\mathrm{LH}_{-} \mathrm{A} 4^{\mathrm{a}}$. & 15 & $2 \times 60$ & $3 \times 120$ & $3 \times 120$ \\
\hline $\mathrm{LH}-\mathrm{A} 5^{\mathrm{a}}$. & 15 & $3 \times 60$ & $6 \times 120$ & $6 \times 120$ \\
\hline $\mathrm{LH}_{-} \mathrm{A} 6^{\mathrm{a}}$. & 15 & $3 \times 60$ & $6 \times 120$ & $6 \times 120$ \\
\hline $\mathrm{LH}_{-} \mathrm{A} 8^{\mathrm{a}} .$. & 15 & $2 \times 60$ & $3 \times 120$ & $3 \times 120$ \\
\hline $\mathrm{LH}_{-} \mathrm{A} 11^{\mathrm{a}}$. & 15 & $5 \times 60$ & $6 \times 120$ & $6 \times 120$ \\
\hline N1_09....... & 15 & $3 \times 60$ & $3 \times 120$ & $3 \times 120$ \\
\hline $\mathrm{N} 2 \_06 \ldots \ldots \ldots \ldots \ldots \ldots \ldots \ldots \ldots \ldots \ldots \ldots$ & 15 & $2 \times 60$ & $2 \times 120$ & $2 \times 120$ \\
\hline 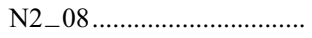 & 15 & $2 \times 60$ & $2 \times 120$ & $2 \times 120$ \\
\hline $\mathrm{N} 2 \_09 \ldots \ldots \ldots \ldots \ldots \ldots \ldots \ldots \ldots \ldots \ldots$ & 15 & $2 \times 60$ & $2 \times 120$ & $2 \times 120$ \\
\hline 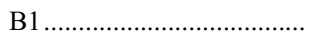 & 12 & $1 \times 240$ & $2 \times 120$ & $2 \times 120$ \\
\hline $\mathrm{B} 2 \ldots \ldots \ldots$ & 15 & $3 \times 60$ & $3 \times 120$ & $3 \times 120$ \\
\hline $\mathrm{B} 3 \ldots \ldots \ldots \ldots$ & 15 & $2 \times 60$ & $2 \times 120$ & $2 \times 120$ \\
\hline 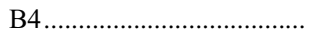 & 15 & $2 \times 60$ & $3 \times 120$ & $3 \times 120$ \\
\hline 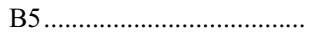 & 15 & $3 \times 60$ & $3 \times 120$ & $3 \times 120$ \\
\hline 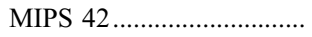 & 3748 & $2 \times 240$ & $2 \times 120$ & $3 \times 120$ \\
\hline MIPS $78 \ldots \ldots \ldots \ldots \ldots \ldots \ldots \ldots \ldots \ldots \ldots \ldots \ldots \ldots \ldots$ & 3748 & $2 \times 240$ & $2 \times 120$ & $3 \times 120$ \\
\hline MIPS $15840 \ldots \ldots \ldots \ldots \ldots \ldots \ldots$ & 3748 & $2 \times 240$ & $6 \times 120$ & $7 \times 120$ \\
\hline 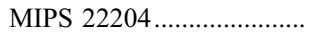 & 3748 & $2 \times 240$ & $2 \times 120$ & $3 \times 120$ \\
\hline MIPS $22303 \ldots \ldots \ldots \ldots \ldots \ldots$ & 3748 & $\ldots$ & $2 \times 120$ & $3 \times 120$ \\
\hline
\end{tabular}

NoTE.-IRS data processed with ver. 13.0 of the SSC pipeline.

${ }^{a}$ IRS data processed with ver. 11.0 of the SSC pipeline.

LL1). The MIPS $24 \mu \mathrm{m}$ fluxes typically agree within $10 \%$ of the $24 \mu \mathrm{m}$ IRS flux.

\section{SPECTRAL PROPERTIES OF THE IRS SPECTRA}

The IRS spectra of all 21 sources are shown in Figure 2. The spectra of an absorbed Seyfert 1 (Mrk 231; Weedman et al. 2005) and a heavily obscured ULIRG (IRAS F00183-7111, hereafter I00183; Tran et al. 2001; Spoon et al. 2004) are also shown for comparison. Both reference spectra exhibit the $\mathrm{Si}$ absorption feature at $9.7 \mu \mathrm{m}$ but with different optical depths, $\tau_{\mathrm{Si}}($ Mrk 231$)=$ 0.65 (Spoon et al. 2002) and $\tau_{\mathrm{Si}}(\mathrm{I} 00183) \geq 5$ (Spoon et al. 2004). The spectrum of I00183 also shows absorption features at 4.26 and $4.67 \mu \mathrm{m}$ due to $\mathrm{CO}_{2}$ and $\mathrm{CO}$ gas and at 6.85 and $7.25 \mu \mathrm{m}$ due to hydrogenated amorphous carbons (HACs; Spoon et al. 2004).

Spectroscopic redshifts are derived from the IRS spectra based on the location of the Si feature in comparison with the spectra of Mrk 231 and I00183. Only in two cases (LH_A4 and B3) are optical spectroscopic redshifts available (Polletta et al. 2006; Desai et al. 2006). For most of the sources, IR spectroscopic redshifts were previously reported (Weedman et al. 2006a; Yan et al. 2007; Houck et al. 2005), although we have revised some of them. The estimated redshifts are reported in Table 3. Based on the comparison with previous $z$ determinations and with the spectroscopic redshifts, we estimate that uncertainties associated with these redshifts are as much as \pm 0.2 for sources with the poorest $\mathrm{S} / \mathrm{N}$.

The $\mathrm{Si}$ feature in absorption is clearly observed in 18 objects (LH_02, LH_A4, LH_A5, LH_A6, LH_A8, LH_A11, N1_09, N2_08, N2_09, B1, B2, B3, B4, B5, MIPS 42, MIPS 78, MIPS 22204, MIPS 22303) and only marginally observed in three objects (LH_01, N2_06, MIPS 15840). The apparent optical depth of the Si feature, $\tau_{\mathrm{Si}}$, is measured as $\ln \left(F_{9.7 \mu \mathrm{m}}^{\text {int }} / F_{9.7 \mu \mathrm{m}}^{\text {obs }}\right)$, where $F_{9.7 \mu \mathrm{m}}^{\mathrm{obs}}$ is the observed flux at $9.7 \mu \mathrm{m}$, at the minimum of the Si feature, and $F_{9.7 \mu \mathrm{m}}^{\text {int }}$ is the intrinsic flux at $9.7 \mu \mathrm{m}$ that would arise from an extrapolated, unabsorbed continuum. Since in most of the cases the SEDs and spectra do not sample the wavelength region beyond the Si feature, we cannot use the standard method to derive the intrinsic continuum (see, e.g., Spoon et al. 2004). The intrinsic flux at $9.7 \mu \mathrm{m}$ is thus estimated using two different methods, (1) by extrapolating a $4-8 \mu \mathrm{m}$ rest-frame power-law fit to $9.7 \mu \mathrm{m}$, and (2) by normalizing an unobscured AGN template to the observed $7.4 \mu \mathrm{m}$ flux, after redshifting it to the redshift of each source, and reddening it using the Galactic center extinction curve (Chiar et al. 2006) until the observed Si feature is well reproduced. The $\mathrm{Si}$ optical depth, $\tau_{\mathrm{Si}}$, is measured only in sources at $z<2.7$ (18 sources). For the remaining we estimate a lower limit since we have only an upper limit to the flux at the bottom of the Si feature. The measured values, reported in Table 3, vary from $>0.2$ to 3.4 .

The expected locations of other spectral features common in starburst galaxies and AGNs are also annotated in Figure 2: (1) absorption features due to molecular gas, $\mathrm{CO}_{2}$, at 4.26 and $4.67 \mu \mathrm{m}$, to water ice at 6.15 and $7.67 \mu \mathrm{m}$, and to HACs at 6.85 and $7.25 \mu \mathrm{m}$; and (2) emission features associated with polycyclic aromatic hydrocarbons (PAHs) at $\lambda=3.3,6.22,7.7,8.6$, and $11.3 \mu \mathrm{m}$, and with low- and high-ionization emission lines, [Ar II] $6.99 \mu \mathrm{m}$, $\mathrm{H}_{2} \mathrm{~S}(3) 9.66 \mu \mathrm{m}$, and [S IV] $10.54 \mu \mathrm{m}$. Absorption features like $\mathrm{CO}$ and HACs would imply the presence of warm gas along the LOS, and features like $\mathrm{CO}_{2}$, water ice, and $\mathrm{Si}$ would imply the presence of shielded cold molecular clouds (Spoon et al. 2004, 2007). Although there are sometimes features in the individual spectra that are consistent with these spectral features, higher $\mathrm{S} / \mathrm{N}$ spectra would be necessary to confirm their detection. No spectrum shows any secure feature except the $\mathrm{Si}$ absorption.

\section{MODELING THE SPECTRAL ENERGY DISTRIBUTIONS WITH TORUS MODELS}

The optical-IR SEDs and rebinned IRS spectra of all 21 sources are shown in Figure 1. The IR SEDs, from 3.6 to $24 \mu \mathrm{m}$, and IRS spectra are modeled with a grid of torus models from Hönig et al. (2006), and the residuals in the NIR are fitted with a galaxy template to represent the host galaxy. The model parameters are the cloud density distribution (from compact to extended), the vertical radial distribution (with various degrees of flaring or nonflaring), the number of clouds along the LOS ( optical depth), and the torus inclination (from face-on to edge-on). The best model is the one that gives the best fit, based on a $\chi^{2}$ test, to the five broadband photometric data points from 3.6 to $24 \mu \mathrm{m}$ and to a maximum of six additional data points derived by interpolating the IRS spectrum at $\lambda=3,5,7,8.5,9.7$, and $12 \mu \mathrm{m}$ in the rest frame.

The best-fit models are shown in Figure 1, and the best-fit parameters are listed in Table 4. In order to fit the stellar component, we adopt a 3 Gyr old elliptical template (from GRASIL; Silva et al. 1998) normalized at the observed flux of the residuals (modelsubtracted SED) at $3.6 \mu \mathrm{m}$ (in the $Z$ band for LH_A5). The choice of an elliptical template is justified by the evidence of ellipticals as hosts in the vast majority of quasars (e.g., Dunlop et al. 2003). In order to check the validity of our assumption on the host galaxy, we estimate the associated $R$-band $\left(\lambda^{\text {rest }}=0.7 \mu \mathrm{m}\right)$ absolute magnitudes, $M_{R}$, and compare them with those measured for the large sample of quasars in Dunlop et al. (2003). The measured $M_{R}$ range from -25.9 to -22.2 , and the median value is -23.7 . These values are consistent with those found by Dunlop et al. (2003), $M_{R}=-23.53 \pm 0.09$, but our sample shows a wider dispersion that can be attributed to the large uncertainty of our method and to the different redshift range. A host galaxy of later type cannot be ruled out, but the low number of detections in the optical, the lack of NIR ( $J H K)$ data, and contamination from the AGN light do not allow us to better constrain the host type. In most of the cases, the elliptical template provides an acceptable fit. 


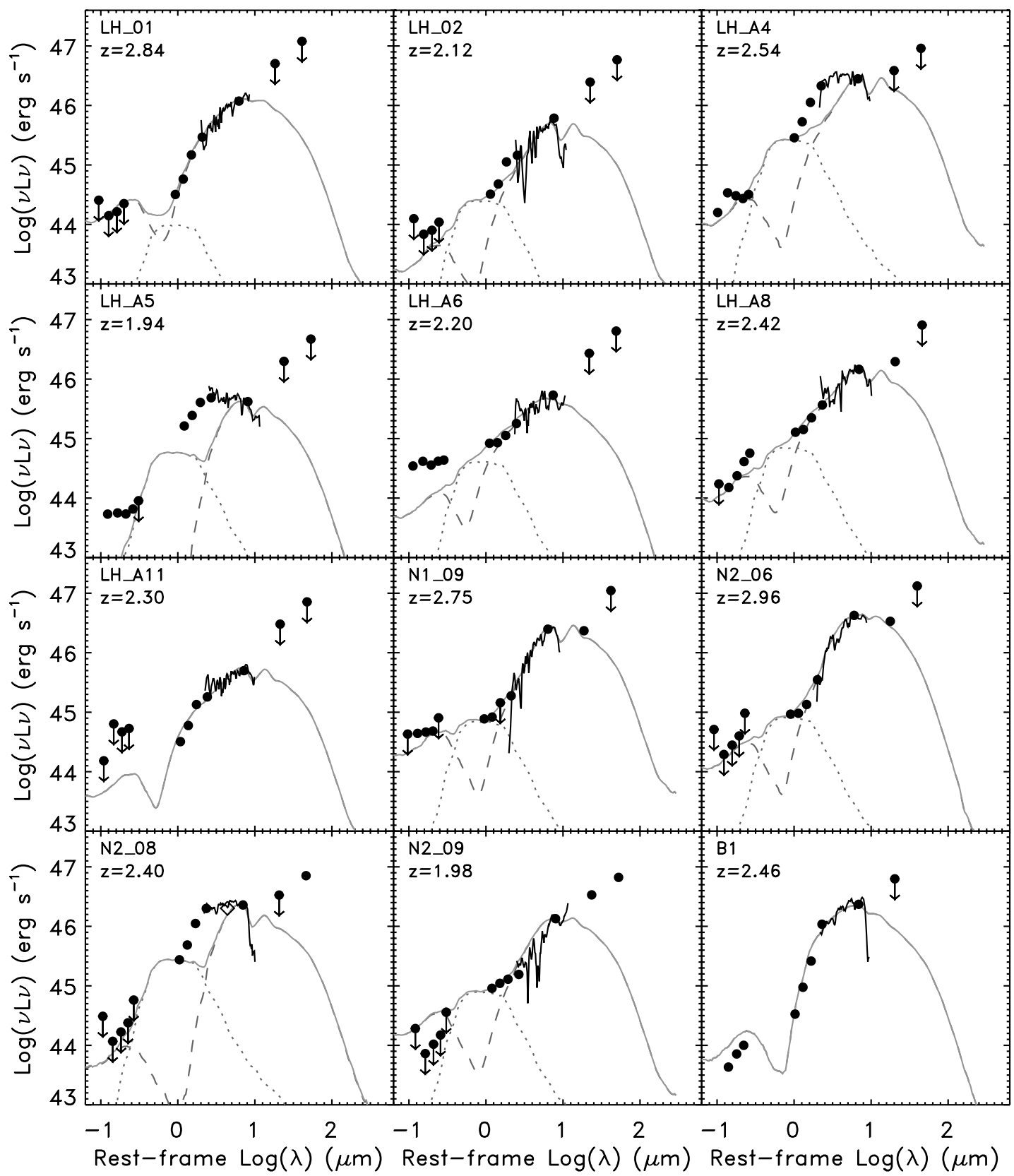

FIG. 1.-Rest-frame SEDs in $\nu L_{\nu}$ vs. $\lambda$ (black symbols and line) and best-fit model (solid gray line) obtained from a torus model (dashed gray line) and an elliptical template (dotted gray line) to fit the residuals in the NIR. Open diamonds represent ISO $15 \mu \mathrm{m}$ (in N2_08) and MAMBO $1.2 \mathrm{~mm}$ (in all E-FLS sources) data (GonzalezSolares et al. 2005; Lutz et al. 2005). Downward-pointing arrows represent $5 \sigma$ upper limits for the optical and infrared data points and $3 \sigma$ upper limits for millimeter data. Source names and redshifts are annotated.

Only in one source, LH_A8, is the SED of the stellar component not well fitted with an elliptical template because of an excess of emission at $\lambda^{\text {rest }}<0.3 \mu \mathrm{m}$ and could a late spiral template provide a better fit. Note that a heavily obscured starburst galaxy would have an optical-NIR SED that is very similar to that of an elliptical galaxy.

The reduced $\chi^{2}$ obtained by comparing the model with the data at $\lambda>1 \mu \mathrm{m}$ in the rest frame are reported in Table 4 . We refer to these as $\mathrm{T}$ (for torus) models (see also Table 4). In 11 cases, a $T$ model well reproduces the observed IR SED and spectrum $\left(\chi_{\nu}^{2}<1\right)$. In the remaining 10 cases, poorer fits are obtained $\left(1<\chi_{\nu}^{2}<10\right)$. In three cases the model underestimates the NIR emission (LH_A4, LH_A5, and N2_08), in four cases the model fails to reproduce the depth of the Si feature (B1, B4, MPS78, and
MIPS 22303), and in the remaining three cases the large $\chi_{\nu}^{2}$ values are caused by either a noisy IRS spectrum (LH_02 and N2_09) or a poor fit at short wavelengths (N2_09 and MIPS 22304). There are also two sources with good $\chi_{\nu}^{2}$ but their fits do not well reproduce the $\mathrm{Si}$ feature. This is due to the fact that the feature is not well sampled by the representative values for the IRS spectrum. There is indeed only one data point in the Si feature.

The main cause of failure in the fits with large $\chi_{\nu}^{2}$ is the inability of the models to reproduce both a prominent NIR emission and a deep Si absorption feature. Yet, sources with deep Si features are expected to have weak NIR emission (e.g., Pier \& Krolik 1992; Levenson et al. 2006). An NIR excess requires that hot dust is seen directly by the observer (typically happening only in less obscured objects), while the Si absorption feature requires 


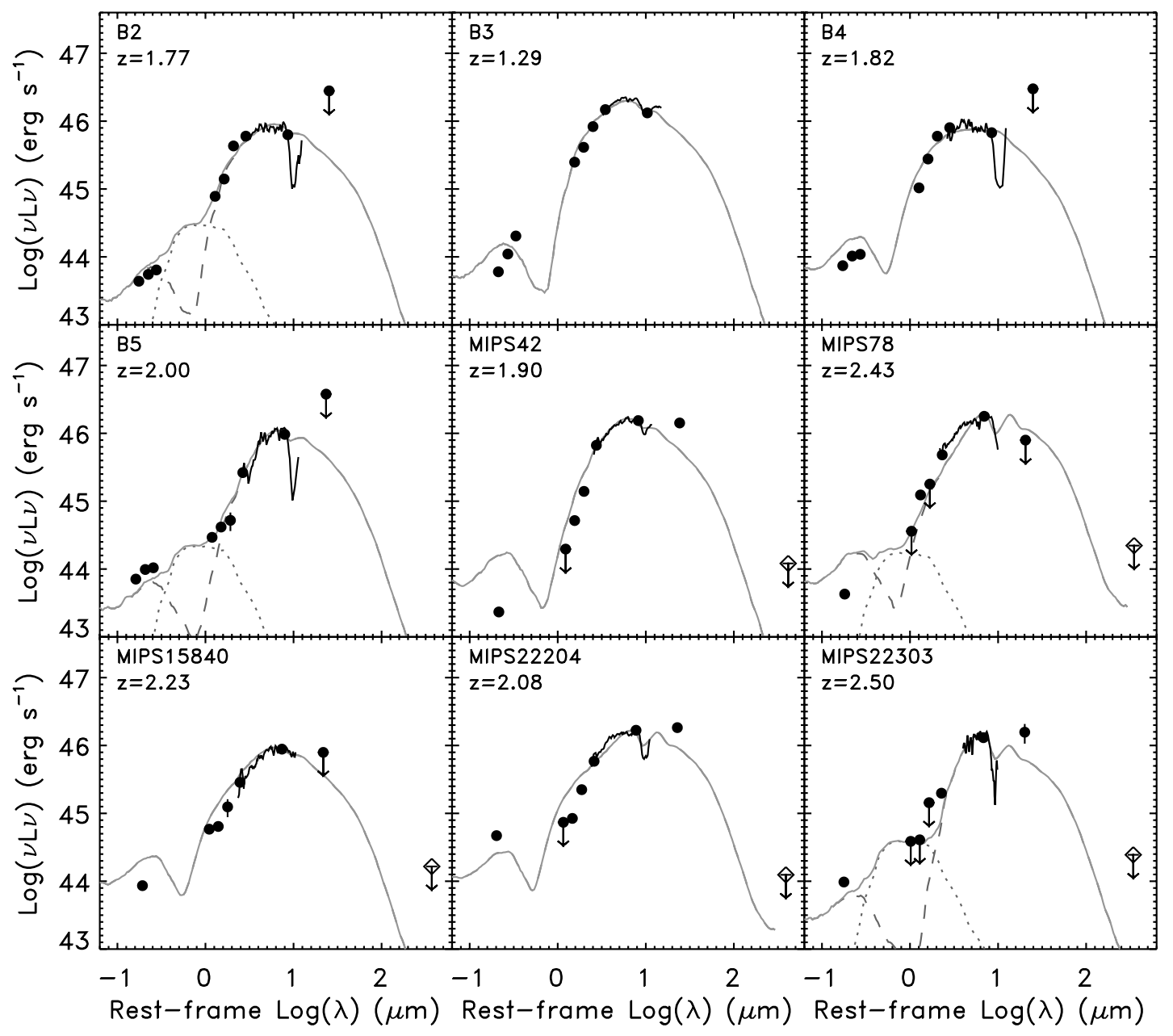

FIG. 1-Continued

the presence of a significant amount of optically and geometrically thick dust (e.g., Pier \& Krolik 1992; Granato et al. 1997; Levenson et al. 2006; Imanishi et al. 2007), and this cooler dust component should absorb the hot dust emission from the vicinity of the obscured AGN.

We attempted, therefore, to model the objects with poor fits, as well as the rest of the sample with an alternative model that includes a cold absorber detached from the torus (hereafter $\mathrm{T}+\mathrm{C}$ models). We assume the Galactic center extinction curve (Chiar et al. 2006) for the cold absorber. We do not model reemission from this absorber for simplicity and because this is expected to occur at far-infrared (FIR) wavelengths. The number of degrees of freedom in the $\chi_{\nu}^{2}$ estimates is 4 in the case of T models and 5 in the case of $\mathrm{T}+\mathrm{C}$ models. The cold absorber is able to produce deeper Si features and significantly improves the fits for nine sources, as shown in Figure 3. In two cases, for B2 and B5, the $\chi_{\nu}^{2}$ have actually increased, but they are still $<1$. In these two sources the $\chi_{\nu}^{2}$ is more sensitive to the broadband photometric data than to the IRS spectrum because of our limited sampling in the observed deep Si feature. But the two fits are equally good, and from a visual inspection of the fits, we choose the model with the cold absorber component as the preferred model because it better fits the $\mathrm{Si}$ feature.

The $\mathrm{T}+\mathrm{C}$ models were adopted for these two sources and for seven sources for which the $\mathrm{T}+\mathrm{C}$ model gives a significantly better fit(LH_A4, LH_A5, N2_08, B1, B2, B4, B5, MIPS 78, and MIPS 22303 ). In two cases ( B4 and N2_08), even the $\mathrm{T}+\mathrm{C}$ model does not provide a good fit to the NIR emission, although it is an improvement with respect to the T model. No improvement was obtained for the three sources with poor $\chi_{\nu}^{2}$ (LH_02, N2_09, and MIPS 22204); therefore, for these we keep the simple $\mathrm{T}$ model. In addition to the $\mathrm{T}+\mathrm{C}$ models, we also show in Figure 3 the torus model before applying the extinction due to the cold absorber (see the dot-dashed line in Fig. 3). The cold absorber can absorb up to $84 \%$ of torus emission in the NIR and MIR $(1-50 \mu \mathrm{m})$, as shown in Figure 3.

In modeling the SEDs, we neglected the optical data. However, we notice that in most of the cases the predicted optical flux from the torus model is lower than the observed optical emission. The optical light might be associated with the host galaxy or with scattered light from the AGN. In six sources detected in the optical, the SED shows an upturn or a blue continuum toward shorter wavelengths, i.e., in the rest-frame far-ultraviolet. This feature can be reproduced by either a population of young stars or emission from the AGN accretion disk. The latter scenario is favored by the optical spectrum of source LH_A4 (aka SW 104409). Its optical spectrum is dominated by a blue faint continuum and narrow emission lines, e.g., Ly $\alpha$ and $\mathrm{C}$ IV $\lambda 1549$ with asymmetric and weak broad components (Polletta et al. 2006). The most likely explanation for the properties of the observed optical spectrum is that it is dominated by scattered light, and the scattering fraction is estimated to be $<1 \%$ (Polletta et al. 2006).

In one case (N2_06), the LOS is at only $30^{\circ}$ from the torus axis, implying an almost clear view of the nuclear region. Since no 

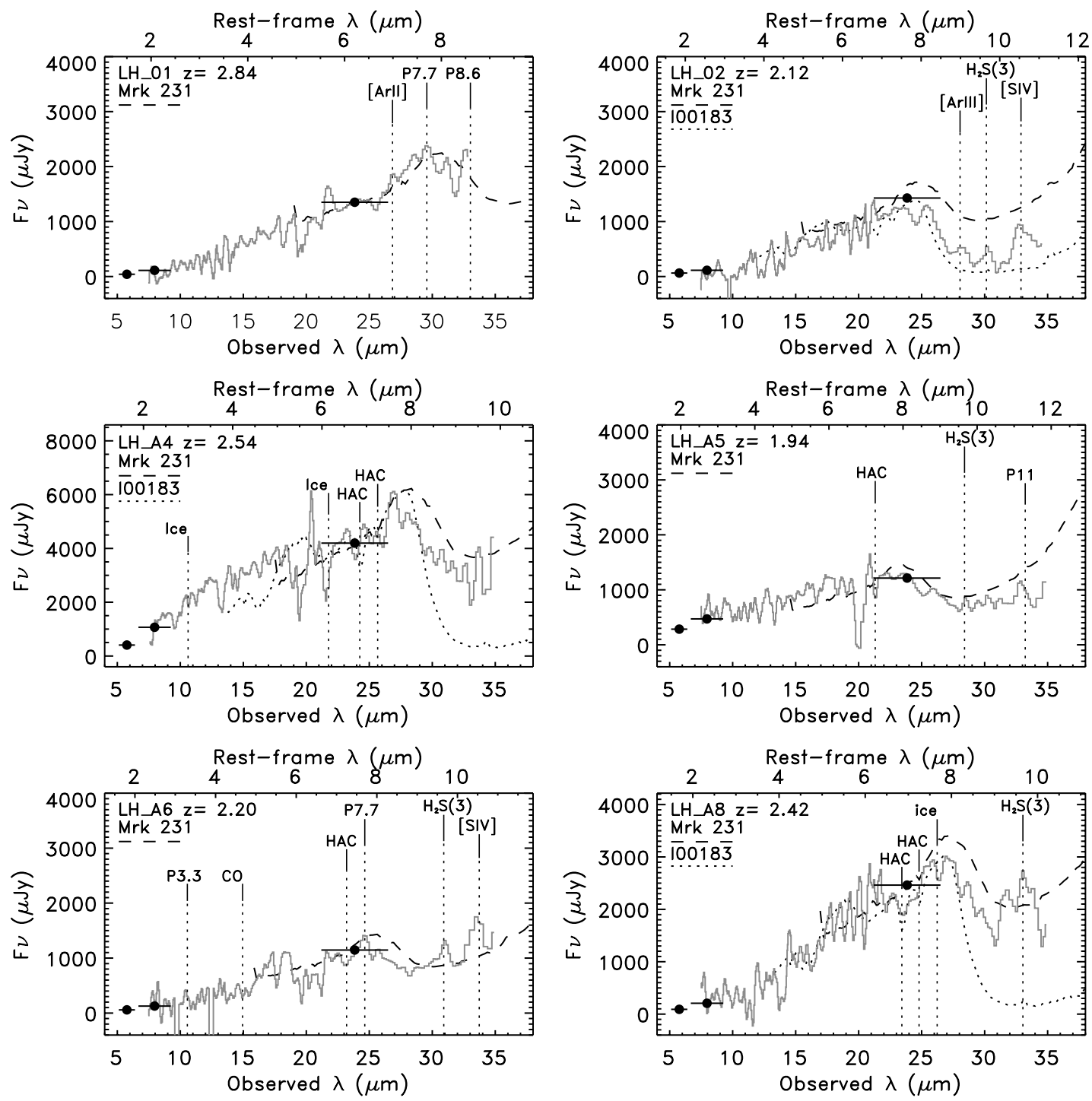

FIG. 2.-Observed IRS spectra (gray solid line) and broadband IR photometric data (black filled circles) of all selected sources. The expected locations of some spectral features are annotated at the corresponding observed wavelength (P refers to PAH emission features). The IRS spectra of the Seyfert 1 Mrk 231 (dotted line) and of the heavily obscured AGN and ULIRG IRAS $000183-7111$ (dashed line) are overplotted for comparison. The name and redshift of each source are annotated, and rest-frame wavelengths are reported on the upper horizontal axis.

additional absorber is required by the data, we expect to see the nuclear emission directly. This seems in apparent contradiction with the faint optical emission of this object. Its best-fit model, however, predicts one optically thick cloud, associated with the torus, along the LOS (see Table 4). The presence of such a cloud in front of the optical source would be enough to absorb the intrinsic optical emission in this source. The estimated apparent Si optical depth in the model is $\tau_{\mathrm{Si}}=0.07-0.34$, which corresponds to a visual optical depth of $0.4-2.1$ or to a suppression factor of the optical flux of $40 \%-0.8 \%$. However, $\tau_{\mathrm{Si}}$ is poorly constrained by the data and in the model because this source is at $z=2.96$. The red MIR SED of this object would imply a much higher optical depth than derived by the Si feature. Because of its high $z$, the dust distribution in N2_06 is thus poorly constrained. A deeper Si might be present, implying a higher torus inclination or the presence of a cold absorber. On the other hand, it is also possible that our best-fit model is correct and that the source has only a weak Si absorption feature and a low-inclination torus, and one optically thick cloud is responsible for suppressing the optical and NIR nuclear light. This scenario might be quite common in obscured QSOs as suggested by a recent study of the IRS spectra of a sample of type 2 absorbed QSOs (Sturm et al. 2006).

\subsection{Model Parameters}

Here we analyze the model parameters of the best-fit models. In the case of the nine sources for which the $\mathrm{T}+\mathrm{C}$ model is preferred, we consider only the parameters obtained with such a model (see Table 4).

The cloud density distribution, which can be considered as an indicator for a compact or extended torus, is expressed by a power law, $n_{r}(r) \propto r^{-a}$, where $r$ is the torus radius. In the models grid, the index $a$ varies from 1 to 3 in steps of 0.5. Larger values of the index $a$ indicate more compact distributions. Since values lower than 1 are not supported by theoretical considerations for an accretion scenario (Beckert \& Duschl 2004), we set a minimum value for the parameter $a$ of unity. In models with $a \geq 1$, the depth of the Si feature increases for larger values of $a$ (see Fig. 10 in Hönig et al. 2006).

Trends of Si strength with $a$ are different for our models based on an accretion torus compared to the models in Levenson et al. (2007). They do not model the dust distribution in a torus but 

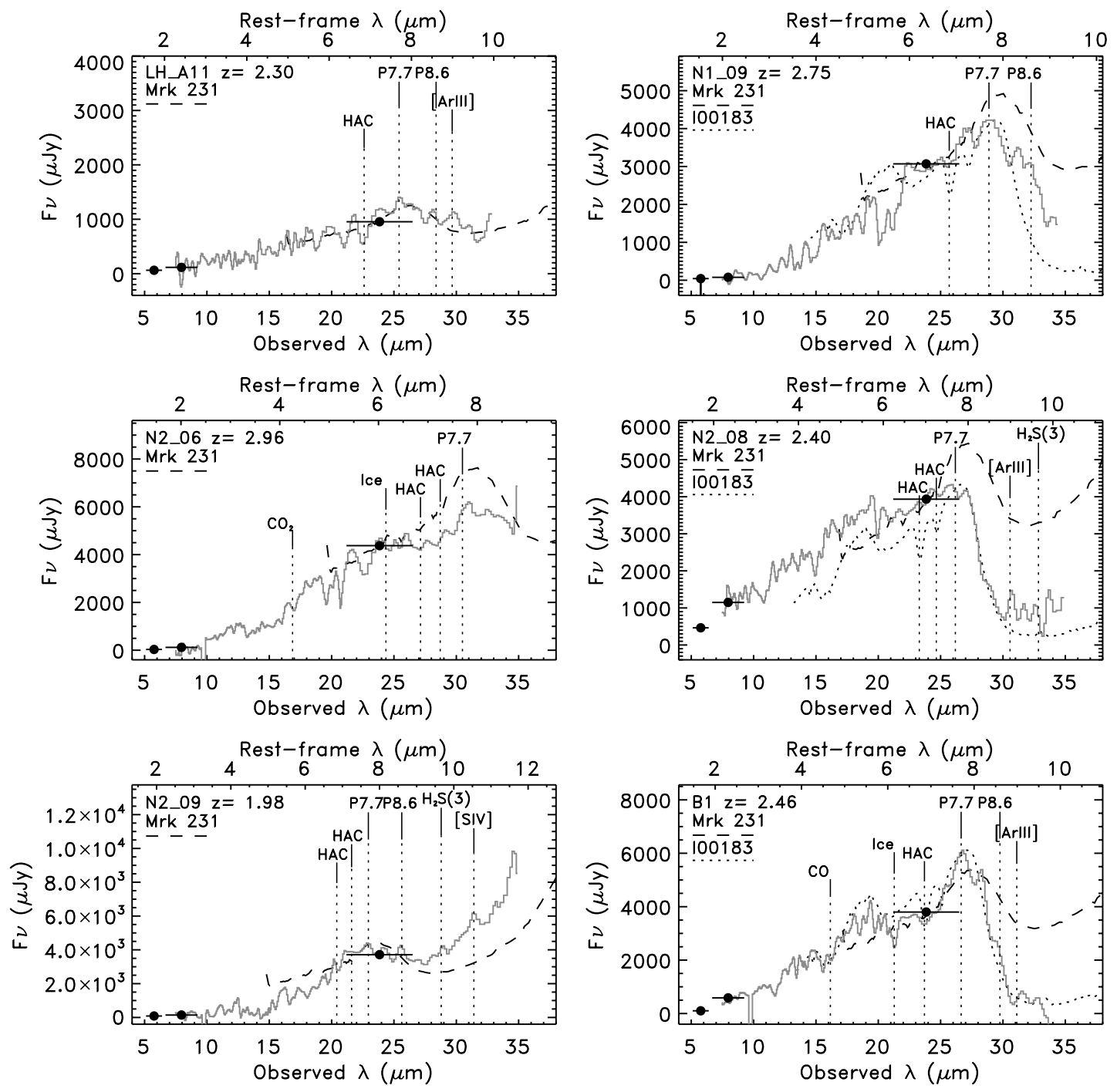

FIG. 2-Continued

instead use different dust distributions (slab and shell) around a central heating source, and they do not consider the combination of a clumpy medium and an additional absorber as proposed here. According to Levenson et al. (2007), the strength of the Si absorption feature is a function of the temperature contrast in dust material, and, thus, a deep absorption feature favors a more extended dust distribution, i.e., $a=0-1$. However, for the majority of our sources, a more compact dust distribution is favored; $a=3$ is chosen in nine cases, $a=2$ in 10 cases, and smaller values, $a \leq 1.5$, are preferred in only two sources.

The clouds' vertical distribution (flaring or nonflaring) is approximated by a power law, $H(r) \propto r^{b}$, where $H$ is the torus scale height and $r$ is the torus radius. In the models grid, the index $b$ can assume values $1,1.5$, and 2 . A nonflaring distribution $(b=1)$ is preferred by 17 sources, and moderately flaring $(b=1.5)$ is preferred only by four sources.

The torus inclination is defined by the angle $\theta$ between the torus axis and the LOS. A face-on torus has $\theta=0^{\circ}$, and an edgeon torus has $\theta=90^{\circ}$. In the models grid, the angle $\theta$ varies from 0 to $90^{\circ}$ in steps of $15^{\circ}$. Our sources do not show a preferred torus inclination; indeed, $\theta$ ranges from $15^{\circ}$ to $90^{\circ}$, with most of the sources $(13)$ at intermediate values $\left(\theta=30^{\circ}-45^{\circ}\right)$. All but two (B5 and MIPS 22303) of the sources modeled with the $\mathrm{T}+\mathrm{C}$ model and one of those with weak Si absorption feature (N2_06) favor a torus with little inclination, $\theta=0^{\circ}-30^{\circ}$. All of the others have inclined tori, $\theta \geq 45^{\circ}$. Therefore, optical obscuration or Si in absorption does not necessarily imply an LOS intercepting the torus (see also Rigby et al. 2006; Brand et al. 2007).

An additional model parameter is the number of clouds along the LOS, $N_{0}^{\mathrm{LOS}}$. This parameter is the average number of clouds obtained from five different cloud arrangements for the same set of model parameters and is thus indicative of the extinction to the AGN emission produced by the torus, $\tau_{V}^{\mathrm{T}} \simeq N_{0}^{\mathrm{LOS}}$ (Natta $\&$ Panagia 1984). The apparent optical depths in the Si feature of the T and $\mathrm{T}+\mathrm{C}$ models, $\tau_{\mathrm{Si}}^{\mathrm{T}}$, and $\tau_{\mathrm{Si}}^{\mathrm{T}+\mathrm{C}}$, are measured following the same procedure applied to the data (see $\S 4)$. The apparent optical depths so derived are reported in Table 4 . The LOS optical depths associated with the torus in the T models are $\tau_{\mathrm{Si}}^{\mathrm{T}} \leq 1.04$, with a median value of $\tau_{\mathrm{Si}}^{\mathrm{T}}=0.13$ or 0.46 depending on the method applied to estimate $\tau_{\mathrm{Si}}^{\mathrm{T}}$. The optical depths associated with the cold absorber are $\tau_{V}^{\mathrm{C}}=4-25$, with a median value of 15 . The apparent optical depths for the sources fitted with the $\mathrm{T}+\mathrm{C}$ model, $\tau_{\mathrm{Si}}^{\mathrm{T}+\mathrm{C}}$, range from 0.6 to 2.9 with a median value of 1.6 or from 0.4 to 1.4 with a median value of 0.9 depending on the method used to measure it.

In Figure 4 we compare the modeled optical depths in the $\mathrm{Si}$ feature, $\tau_{\mathrm{Si}}^{\text {mod }}$, with the observed optical depths, $\tau_{\mathrm{Si}}^{\text {meas }}$ (see $\S 4$ ). The modeled optical depths range from 0.01 to 2.9 , considering 

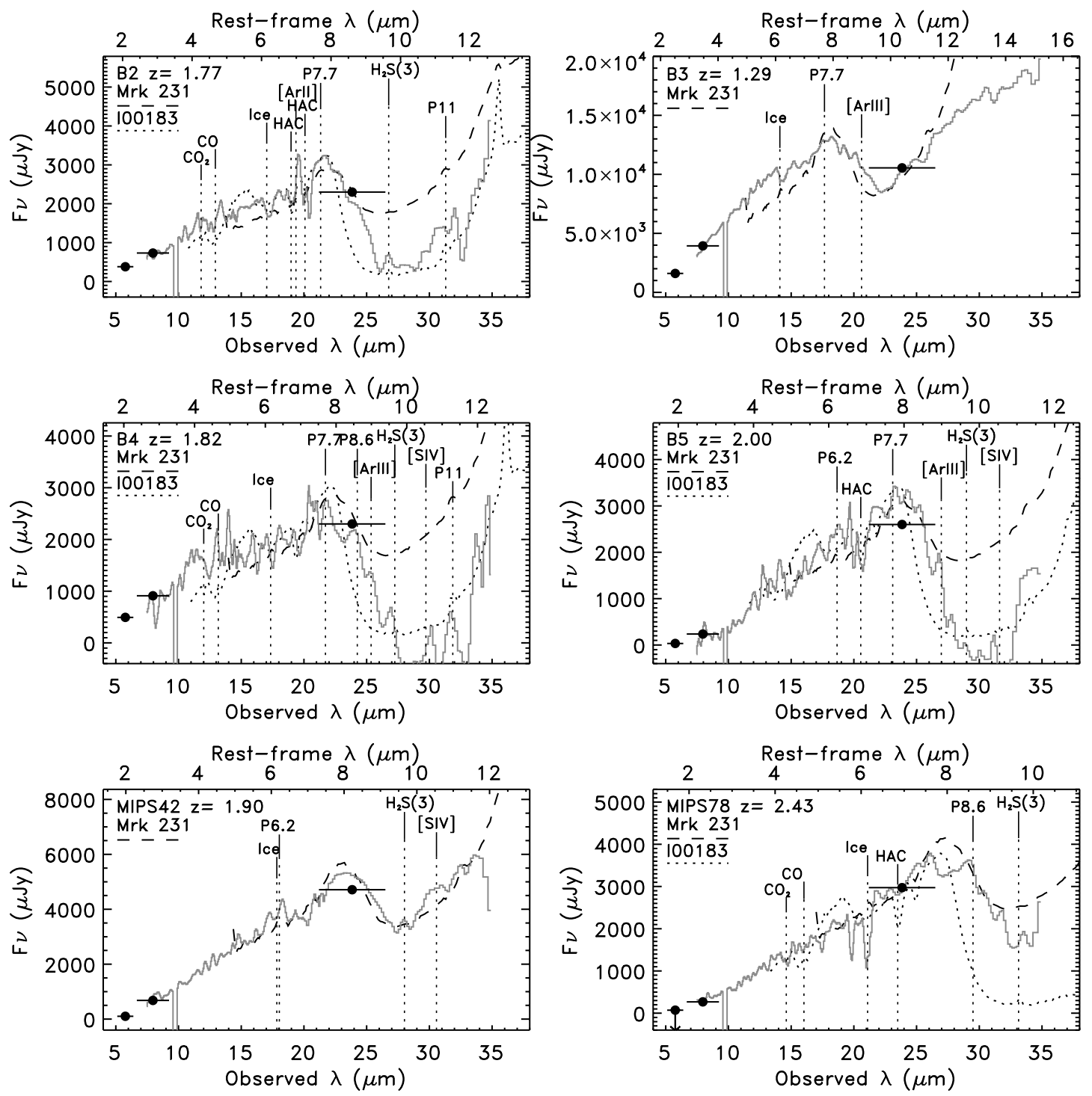

FIG. 2-Continued

both the $\mathrm{T}$ and $\mathrm{T}+\mathrm{C}$ models. The modeled and measured $\tau_{\mathrm{Si}}$ satisfy the following relation: $\tau_{\mathrm{Si}}^{\text {mod }}=(0.96 \pm 0.13) \tau_{\mathrm{Si}}^{\text {meas }}-0.03 \pm 0.13$. Although there is a general agreement between the two estimates, it is clear that these kinds of measurements are characterized by large uncertainties, especially in objects at high redshift for which the Si feature is not well sampled, and that it is a difficult task to model both the NIR-MIR continuum and the Si absorption feature.

In summary, we find that there is no preferred torus inclination associated with the detection of a Si feature in absorption. However, in the cases where the absorption feature is well detected and can be modeled by the torus model, the inclination is always higher than $\theta=45^{\circ}$, consistent with the LOS intercepting the torus. Sources with a less inclined torus require an additional absorber to explain the observed Si feature or show a weak Si feature. A compact nonflaring torus is preferred by the majority of the sources. The clouds' radial density distribution indeed indicates that the torus emission region is compact, or that the NIR and MIR emission is dominated by dust in the vicinity of the nucleus. The preferred nonflaring torus in the majority of these luminous sources is consistent with the predictions of the receding torus models (e.g., Simpson 2005; Hönig \& Beckert 2007). According to these models, the opening angle of the torus increases at larger luminosities. Our results are in agreement with the predictions from Hönig \& Beckert (2007). These authors claim that flaring should not occur in high-luminosity sources because large clouds at large distances from the AGN should be driven away by the radiation pressure.

\subsection{Far-IR Emission}

According to current AGN evolutionary models, obscured and extremely luminous AGNs are believed to represent a specific and rare phase in the evolution of an AGN, when the central supermassive black hole (SMBH) is fully grown and still surrounded by a large amount of dust and gas (Sanders et al. 1988; Di Matteo et al. 2005; Hopkins et al. 2005). High-luminosity AGNs are believed to be triggered by large-scale galaxy mergers and, therefore, to be accompanied by intense starburst activity. In order to test whether this scenario applies to our sample, we search for starburst signatures in our objects. Obscured starburst galaxies are generally heavily extincted at optical wavelengths and are weak X-ray sources compared to AGNs. Thus, the only observations that could reveal a starburst are PAH features at MIR wavelengths and strong continuum from cool dust at FIR and submillimeter 

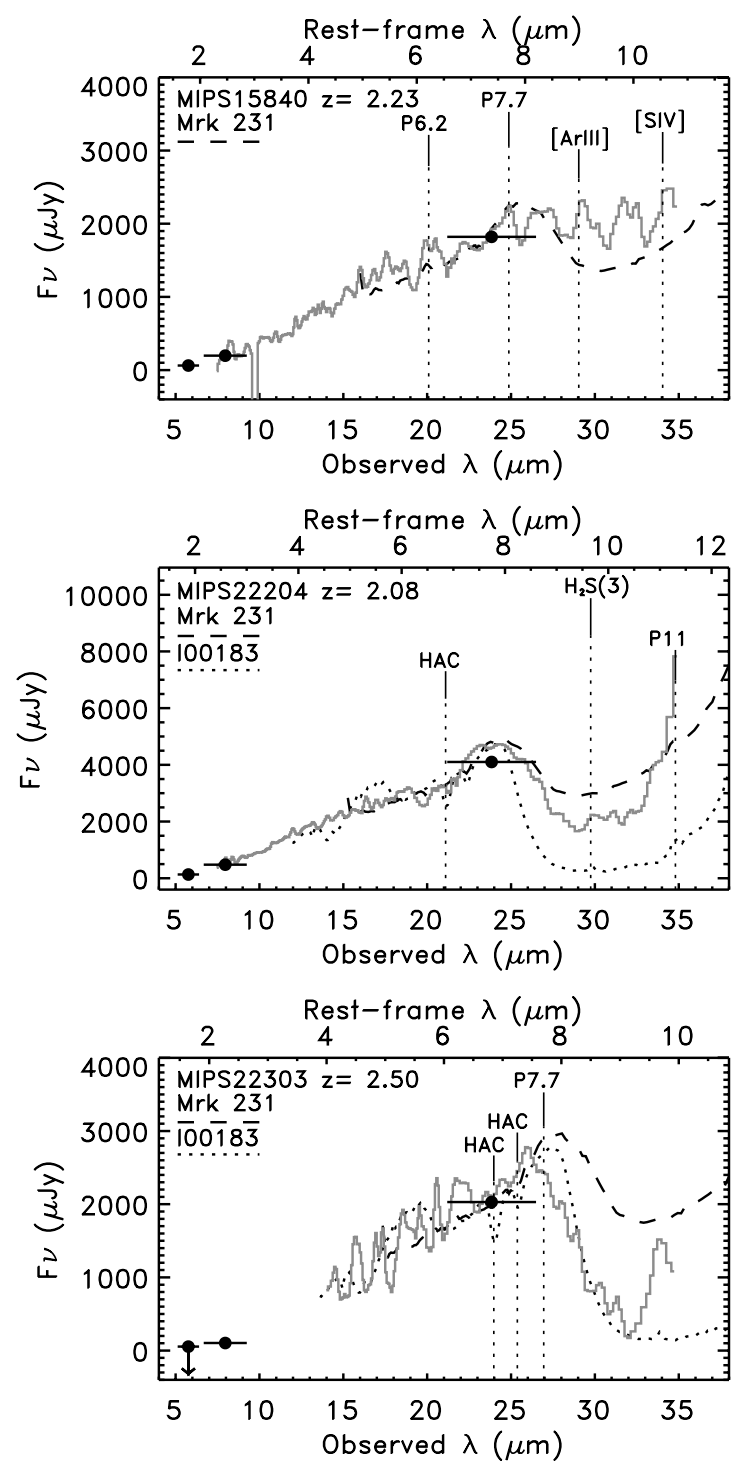

FIG. 2-Continued

wavelengths. An analysis of the starburst contribution based on the PAH features is discussed in $\S 9.1$; here we analyze the FIR properties.

Most of the power produced by a starburst emerges in the FIR. The typical FIR emission of powerful starbursts is characterized by luminosities $\simeq 10^{11.0}-10^{12.5} L_{\odot}$ and peaks at $60-100 \mu \mathrm{m}$ (Sanders \& Mirabel 1996). Such luminosities correspond to star formation rates (SFRs) of $\sim 20-550 M_{\odot} \mathrm{yr}^{-1}$ (Kennicutt 1998). Higher rates, up to $\sim 5000 M_{\odot} \mathrm{yr}^{-1}$, and luminosities, up to $3 \times$ $10^{13} L_{\odot}$, are measured in some high- $z$ ULIRGs/submillimeter galaxies (SMGs) and attributed to starbursts (e.g., Chapman et al. 2004).

In our sample, five SWIRE sources are detected at 70 or $160 \mu \mathrm{m}$ and three E-FLS sources are detected at $70 \mu \mathrm{m}$. Note that both MIPS FIR observations are available only for SWIRE sources (13 sources), and MIPS $70 \mu \mathrm{m}$ pointed observations are available for the E-FLS sources (Sajina et al. 2007a). The SWIRE observations in the FIR are sensitive enough only to reveal FIR luminosities $>10^{13} L_{\odot}$ at the observed redshifts (the $5 \sigma$ limits are 18 and $108 \mathrm{mJy}$ at 70 and $160 \mu \mathrm{m}$, respectively). In Figure 5 we compare the SWIRE $5 \sigma$ detection limit and the detected fluxes at 70 and $160 \mu \mathrm{m}$ of our sources with those expected for two starburst galaxies, M82 and Arp 220, with an IR luminosity of $10^{11}$ and $10^{12.5} L_{\odot}$ at $1<z<3.2$. The figure clearly shows that we do not expect to detect the FIR emission of starbursts with such luminosities at $z>1.2$.

What is then the origin of the detected FIR emission in our objects? We investigate three possible origins: the torus, AGNheated dust at large distances from the nucleus, and an exceptionally powerful starburst. The spectrum of our torus models peaks at around $10 \mu \mathrm{m}$, corresponding to a temperature of about $300 \mathrm{~K}$, and falls off at longer wavelengths. In two cases, the $70 \mu \mathrm{m}$ detections or upper limits agree well with our models (N1_09 and N2_06), but in the other six cases, the observed 70 or $160 \mu \mathrm{m}$ fluxes are significantly higher than the model predictions ( $2 H \_A 8$, N2_08, N2_09, MIPS 42, MIPS 22204, and MIPS 22303) and require an additional component to be explained. The SEDs of these sources resemble that of the starburst/AGN composite source CXO J1417 discussed in Le Floc'h et al. (2007). The FIR luminosity and SFR estimated for CXO J1417 are $4.5 \times 10^{12} L_{\odot}$ and $\sim 750 M_{\odot} \mathrm{yr}^{-1}$, respectively, as in a powerful starburst.

In order to quantify the amount of luminosity detected in the FIR, we model the observed fluxes at rest frame $\lambda>5 \mu \mathrm{m}$ with a starburst template. We first derive the residual fluxes after subtracting 
TABLE 3

Infrared Luminosities and Optical Depths

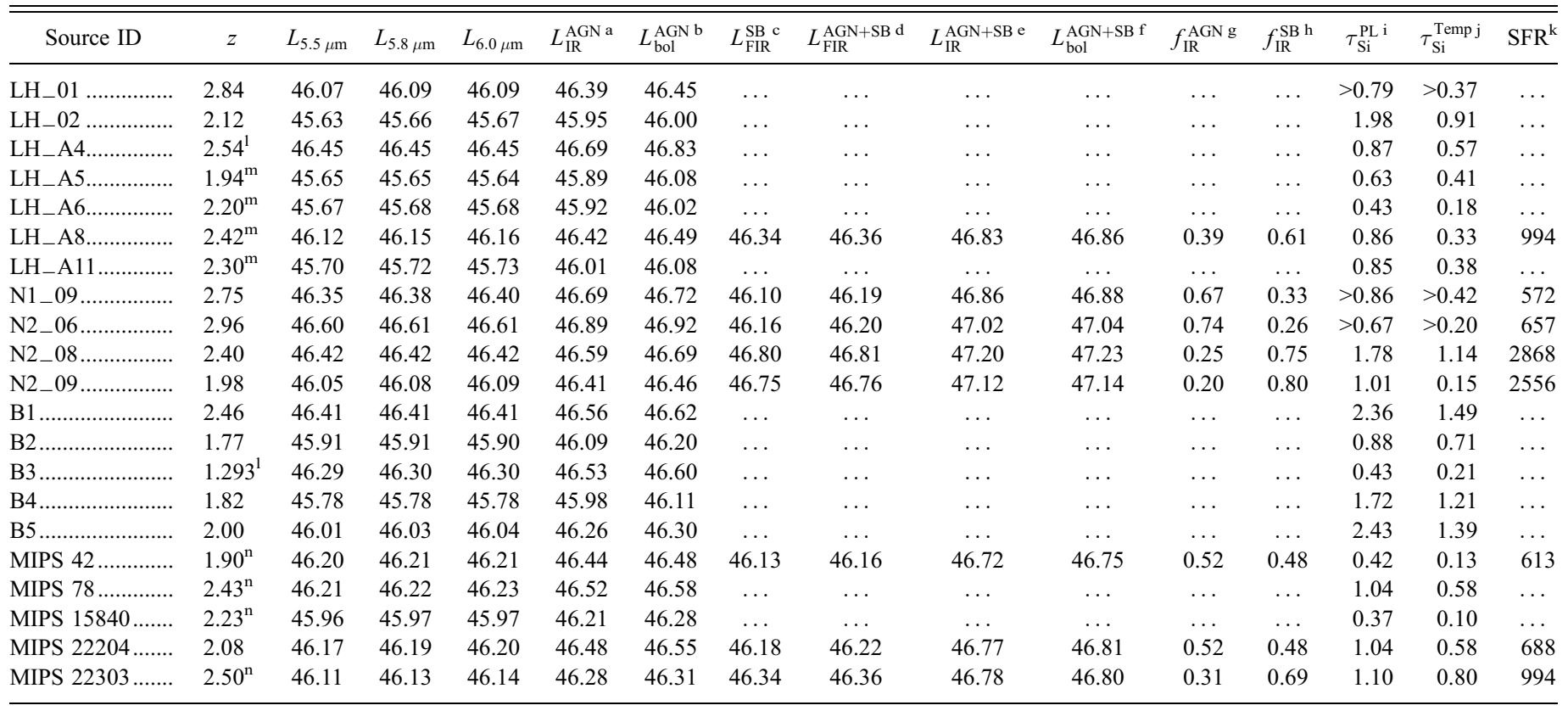

Notes.-All luminosities are logarithm in units of ergs s ${ }^{-1}$. Luminosities for a starburst component are estimated only for sources detected at 70 or $160 \mu \mathrm{m}$.

${ }^{a}$ Logarithm of the AGN IR luminosity obtained by integrating the torus model between 3 and $1000 \mu \mathrm{m}$.

${ }^{\mathrm{b}}$ Logarithm of the AGN bolometric luminosity obtained by integrating the torus model between 0.1 and $1000 \mu \mathrm{m}$.

${ }^{c}$ Logarithm of the starburst component FIR luminosity between 42.5 and $122.5 \mu \mathrm{m}$.

${ }^{\mathrm{d}}$ Logarithm of the AGN and starburst FIR luminosities between 42.5 and $122.5 \mu \mathrm{m}$.

e Logarithm of the AGN and starburst IR luminosities between 3 and $1000 \mu \mathrm{m}$.

${ }^{\mathrm{f}}$ Logarithm of the AGN and starburst bolometric luminosities.

graction of AGN luminosity to the total IR luminosity.

${ }^{\text {h }}$ Fraction of starburst luminosity to the total IR luminosity.

Silicate optical depth derived from $\ln \left(F_{9.7 \mu \mathrm{m}}^{\text {int }} / F_{9.7 \mu \mathrm{m}}^{\text {obs }}\right)$ with $F_{9.7 \mu \mathrm{m}}^{\text {int }}$ estimated extrapolating a power-law model fit to the data at $\lambda<7 \mu \mathrm{m}$ in the rest frame.

${ }^{j}$ Silicate optical depth derived from $\ln \left(F_{9.7}^{\text {int }} \mu \mathrm{m} / F_{9.7}^{\text {obs }} \mu \mathrm{m}\right)$ with $F_{9.7}^{\text {int }} \mu \mathrm{m}$ estimated from a type 1 QSO template normalized at the observed $24 \mu \mathrm{m}$ flux and redshifted at the redshift of the source.

${ }^{\mathrm{k}}$ Star formation rate derived from $L_{\mathrm{FIR}}^{\mathrm{SB}}$ using the Kennicutt (1998) relationship.

${ }^{1}$ Optical spectroscopic redshift for LH_A4 from Polletta et al. (2006) and for B3 from Desai et al. (2006).

${ }^{m}$ LH_A5: $z=1.89$; LH_A6: $z=2.10$; LH_A8: $z=2.31$; LH_A11: $z=2.25$; in Weedman et al. (2006a).

${ }^{n}$ MIPS 42: $z=1.95 \pm 0.07$; MIPS 78: $z=2.65 \pm 0.1$; MIPS 15840: $z=2.3 \pm 0.1$; MIPS 22303: $z=2.34 \pm 0.14$; in Yan et al. (2007).

the torus contribution to the observed FIR fluxes and then fit them with starburst templates. Two different starburst templates, M82 and Arp 220 (Silva et al. 1998), are used for the fits, but we only show the results obtained with the M82 template because it provides better fits in the majority of the cases and similar good fits in the remaining cases. The starburst fits, combined with the torus and host galaxy fits, are shown in Figure 6. The estimated starburst luminosities and contribution to the total IR luminosity are reported in Table 3. In half of the FIR-detected sources the torus contribution to the total IR luminosity is lower than that of the additional FIR component. The remaining objects divide equally between those for which the two contributions are similar and those for which the torus contribution is larger. The estimated starburst FIR luminosities are all greater than $3.3 \times 10^{12} L_{\odot}$. These luminosities imply SFRs $>600 M_{\odot} \mathrm{yr}^{-1}$ (Kennicutt 1998), which are consistent only with those measured in the most powerful starbursts.

Another possible origin of the FIR component is reemission of the energy absorbed by the cold absorber discussed in $\S 5$. There are two sources detected at long wavelengths and modeled with the T+C model, N2_08 and MIPS 22303. The estimated absorbed luminosities in the $1-50 \mu \mathrm{m}$ wavelength range are $1.8 \times 10^{47}$ and $5.25 \times 10^{45} \mathrm{ergs} \mathrm{s}^{-1}$ for N2_08 and MIPS 22303, respectively. These correspond to 2.8 and 0.24 times the FIR luminosities derived from the starburst template $\left(L_{\mathrm{FIR}}^{\mathrm{SB}}\right)$. Although these ratios depend on the cold absorber covering factor and on the efficiency in reemitting the absorbed energy, these values indicate that this hypothesis is energetically viable.

Although we can reproduce the FIR component with a starburst template or with reemission from the cold absorber, its origin remains undetermined. Large FIR luminosities, as measured in our sources, even when cold molecular gas is detected, do not probe the presence of a powerful starburst, e.g., in QSOs where [O II] 23727 emission is not detected (Ho 2005). Thus, both explanations remain plausible and do not exclude others. In order to understand the origin of the FIR component, we would need more FIR measurements, higher S/N IR spectra, and NIR spectra. Multiple FIR detections would help to constrain the radiation field, high-S/N IR spectra would allow us to search for and, eventually, measure PAH emission (see $\S 9.1$ ), and NIR spectra could be used to constrain the SFR from the [O II] $\lambda 3727$ emission line.

\subsection{AGN Bolometric Luminosity}

Assuming the best-fit torus model and integrating it from $1000 \AA$ to $1000 \mu \mathrm{m}$, we can derive a lower limit to the AGN bolometric luminosity. This estimate of AGN bolometric luminosity does not include the AGN optical light that is not reprocessed by the 
TABLE 4

Torus Model Parameters

\begin{tabular}{|c|c|c|c|c|c|c|c|c|c|c|}
\hline Source ID & Model $^{\mathrm{a}}$ & $a^{\mathrm{b}}$ & $b^{\mathrm{c}}$ & $\begin{array}{c}\theta^{\mathrm{d}} \\
(\mathrm{deg})\end{array}$ & $N_{0}{ }^{\mathrm{e}}$ & $N_{0}^{\mathrm{LOS} \mathrm{f}}$ & $\tau_{V}^{\mathrm{CA} g}$ & $\tau_{\mathrm{Si}}^{\mathrm{PL} \mathrm{h}}$ & $\tau_{\mathrm{Si}}^{\mathrm{Temp} \mathrm{h}}$ & $\chi_{\nu}^{2 \mathrm{i}}$ \\
\hline LH_01 ..................... & $\mathrm{T}$ & 3.0 & 1.5 & 60 & 12 & 6.0 & 0 & 0.29 & 0.01 & 0.016 \\
\hline LH_02 .................... & $\mathrm{T}$ & 3.0 & 1.0 & 90 & 26 & 26.0 & 0 & 1.00 & 0.32 & 5.085 \\
\hline \multirow[t]{2}{*}{ LH_A4 ..................... } & $\mathrm{T}$ & 3.0 & 1.0 & 60 & 12 & 9.0 & 0 & 1.00 & 0.32 & 4.638 \\
\hline & $\mathrm{T}+\mathrm{C}$ & 2.0 & 1.0 & 15 & 26 & 0.0 & 6 & 0.62 & 0.35 & 0.575 \\
\hline \multirow[t]{2}{*}{ LH_A5 ...................... } & $\mathrm{T}$ & 1.5 & 1.5 & 60 & 18 & 17.5 & 0 & 0.83 & 0.27 & 6.665 \\
\hline & $\mathrm{T}+\mathrm{C}$ & 2.0 & 1.0 & 30 & 26 & 0.2 & 7 & 0.62 & 0.36 & 0.703 \\
\hline LH_A6.................. & $\mathrm{T}$ & 2.0 & 1.0 & 45 & 26 & 5.6 & 0 & 0.32 & 0.07 & 0.050 \\
\hline LH_A $8 \ldots \ldots \ldots \ldots \ldots \ldots . . . . . . .$. & $\mathrm{T}$ & 3.0 & 1.0 & 60 & 9 & 6.5 & 0 & 0.81 & 0.26 & 0.078 \\
\hline $\mathrm{LH}_{-} \mathrm{A} 11 \ldots \ldots \ldots \ldots \ldots \ldots$ & $\mathrm{T}$ & 3.0 & 1.0 & 45 & 12 & 4.6 & 0 & 0.75 & 0.25 & 0.533 \\
\hline 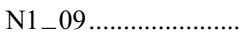 & $\mathrm{T}$ & 3.0 & 1.0 & 60 & 12 & 9.0 & 0 & 1.04 & 0.29 & 0.021 \\
\hline N2_06 ....................... & $\mathrm{T}$ & 1.1 & 1.5 & 30 & 13 & 1.0 & 0 & 0.34 & 0.06 & 0.004 \\
\hline \multirow[t]{2}{*}{ N2_08 ..................... } & $\mathrm{T}$ & 2.0 & 1.0 & 90 & 26 & 26.0 & 0 & 1.05 & 0.40 & 10.120 \\
\hline & $\mathrm{T}+\mathrm{C}$ & 2.0 & 1.0 & 30 & 26 & 0.2 & 21 & 2.33 & 1.35 & 2.515 \\
\hline N2_-09..................... & $\mathrm{T}$ & 3.0 & 1.5 & 45 & 8 & 5.2 & 0 & 0.49 & 0.04 & 2.307 \\
\hline \multirow[t]{2}{*}{ 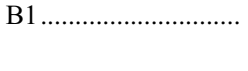 } & $\mathrm{T}$ & 2.0 & 1.0 & 45 & 26 & 5.6 & 0 & 0.37 & 0.11 & 1.435 \\
\hline & $\mathrm{T}+\mathrm{C}$ & 3.0 & 1.0 & 15 & 26 & 0.0 & 25 & 2.94 & 1.64 & 0.042 \\
\hline \multirow[t]{2}{*}{ B2 $\ldots \ldots \ldots \ldots \ldots \ldots \ldots \ldots \ldots \ldots \ldots \ldots \ldots \ldots$} & $\mathrm{T}$ & 2.0 & 1.0 & 45 & 26 & 5.6 & 0 & 0.37 & 0.12 & 0.391 \\
\hline & $\mathrm{T}+\mathrm{C}$ & 2.0 & 1.0 & 30 & 26 & 0.2 & 18 & 1.85 & 1.07 & 0.540 \\
\hline 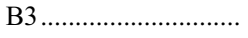 & $\mathrm{T}$ & 2.0 & 1.0 & 45 & 26 & 5.6 & 0 & 0.37 & 0.11 & 0.530 \\
\hline \multirow[t]{2}{*}{ 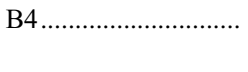 } & $\mathrm{T}$ & 2.0 & 1.0 & 30 & 26 & 0.2 & 0 & 0.01 & 0.00 & 10.000 \\
\hline & $\mathrm{T}+\mathrm{C}$ & 2.0 & 1.0 & 30 & 26 & 0.2 & 16 & 1.60 & 0.92 & 4.898 \\
\hline \multirow[t]{2}{*}{ 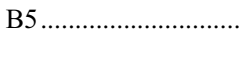 } & $\mathrm{T}$ & 2.0 & 1.0 & 45 & 26 & 5.6 & 0 & 0.56 & 0.14 & 0.006 \\
\hline & $\mathrm{T}+\mathrm{C}$ & 1.5 & 1.5 & 90 & 6 & 6.0 & 15 & 2.02 & 0.93 & 0.295 \\
\hline MIPS $42 \ldots \ldots \ldots \ldots \ldots \ldots$ & $\mathrm{T}$ & 2.0 & 1.0 & 45 & 26 & 5.6 & 0 & 0.43 & 0.14 & 0.390 \\
\hline \multirow[t]{2}{*}{ MIPS $78 \ldots \ldots \ldots \ldots \ldots \ldots$} & $\mathrm{T}$ & 3.0 & 1.0 & 60 & 12 & 4.6 & 0 & 1.00 & 0.31 & 2.153 \\
\hline & $\mathrm{T}+\mathrm{C}$ & 3.0 & 1.0 & 30 & 9 & 0.3 & 10 & 0.93 & 0.40 & 0.003 \\
\hline MIPS $15840 \ldots \ldots \ldots \ldots . . .$. & $\mathrm{T}$ & 2.0 & 1.0 & 45 & 26 & 5.6 & 0 & 0.32 & 0.07 & 0.155 \\
\hline MIPS $22204 \ldots \ldots \ldots \ldots . . .$. & $\mathrm{T}$ & 3.0 & 1.0 & 45 & 12 & 4.6 & 0 & 0.75 & 0.25 & 2.957 \\
\hline \multirow[t]{2}{*}{ MIPS $22303 \ldots \ldots \ldots \ldots$} & $\mathrm{T}$ & 2.0 & 1.0 & 90 & 26 & 26.0 & 0 & 1.06 & 0.40 & 2.438 \\
\hline & $\mathrm{T}+\mathrm{C}$ & 2.0 & 1.0 & 90 & 26 & 26.0 & 4 & 1.54 & 0.68 & 0.775 \\
\hline
\end{tabular}

a $\mathrm{T}$ : torus model; $\mathrm{T}+\mathrm{C}$ : torus + cold absorber model. The parameters for the $\mathrm{T}+\mathrm{C}$ model are given only when the torus-only $(\mathrm{T})$ model gives a poor fit $\left(\chi_{\nu}^{2}>0.1\right)$ to the observed SED and spectrum, or the T+C model significantly improves the $\chi^{2}$.

b Index of the torus density radial distribution, $n_{r}(r) \propto r^{-a}$, where $r$ is the torus radius.

c Index of the clouds' vertical distribution, $H \propto r^{b}$, where $H$ is the torus scale height.

d Inclination of the torus axis with respect to the LOS.

e Average number of clouds along an equatorial LOS.

f Average number of clouds along the LOS, which approximately corresponds to the optical depth of the torus in the visual (Natta \& Panagia 1984).

g Cold absorber (CA) optical depth in the visible.

h Absorber (torus for model T and torus + cold absorber for model $\mathrm{T}+\mathrm{C}$ ) optical depth associated with the Si feature measured by extrapolating a powerlaw model $\left(\tau_{\mathrm{Si}}^{\mathrm{PL}}\right)$ or by fitting a QSO template $\left(\tau_{\mathrm{Si}}^{\mathrm{Temp}}\right)$ (see text).

${ }^{\mathrm{i}}$ Reduced $\chi^{2}$ obtained from the best-fit model and the observed data at $\lambda>1 \mu \mathrm{m}$ in the rest frame.

dust, and it is not emitted along our LOS. Thus, the true AGN bolometric luminosity should be corrected by a factor that depends on the torus covering factor and anisotropy of the torus reemission. The covering factor of a torus can vary from $20 \%$ to $50 \%$, implying AGN bolometric luminosities that can be $2-5$ times larger than our estimates. The uncertainty associated with the redshift estimates $( \pm 0.2)$ corresponds to a $30 \%(20 \%)$ uncertainty in the luminosity estimate for objects at $z \simeq 2(z \simeq 3)$. We do not include the luminosity associated with the host galaxy. Note that the discrepancy between the observed optical data and the best-fit models does not affect the results, since the bulk of the luminosity is emitted around $10 \mu \mathrm{m}$ in the rest frame, where the SED is well reproduced by the models. The estimated bolometric luminosities, reported in Table 3, range from $10^{46}$ to $10^{47} \mathrm{ergs} \mathrm{s}^{-1}$.

The estimated AGN bolometric luminosities are compared with the $6 \mu \mathrm{m}$ luminosities in Figure 7 in order to investigate whether it is possible to determine AGN bolometric luminosities from the observed $6 \mu \mathrm{m}$ luminosities without modeling the SED and without having measurements at long wavelengths. We find that the two luminosities are highly correlated and that the AGN bolo- metric luminosity can be derived from the $6 \mu \mathrm{m}$ luminosity adopting the following relationship:

$$
\log L_{\mathrm{bol}}^{\mathrm{AGN}}=\log L(6 \mu \mathrm{m})+0.32 \pm 0.06 .
$$

This relationship gives a bolometric luminosity that is 7 times smaller than what would be derived assuming the bolometric corrections obtained for a sample of optically selected, type 1 QSOs by Elvis et al. (1994), $\log L_{\text {bol }}=\log L(6 \mu \mathrm{m})+1.16$. Such a difference is mainly due to the fact that $L_{\text {bol }}$ in Elvis et al. (1994) includes both the AGN optical emission that is not included in our analysis and the emission from the host galaxy. Their $L_{\mathrm{bol}}$ also includes a significant contribution in the FIR from cool dust that is not included in our models and thus in our estimates of $L_{\text {bol }}^{\mathrm{AGN}}$. Moreover, their QSOs are about 100 times less MIR luminous than our sample, and, thus, it is also probable that their median template does not well represent the MIR-FIR SEDs of our objects.

For the eight objects for which FIR data are available (see previous section), we also estimate a bolometric luminosity including 


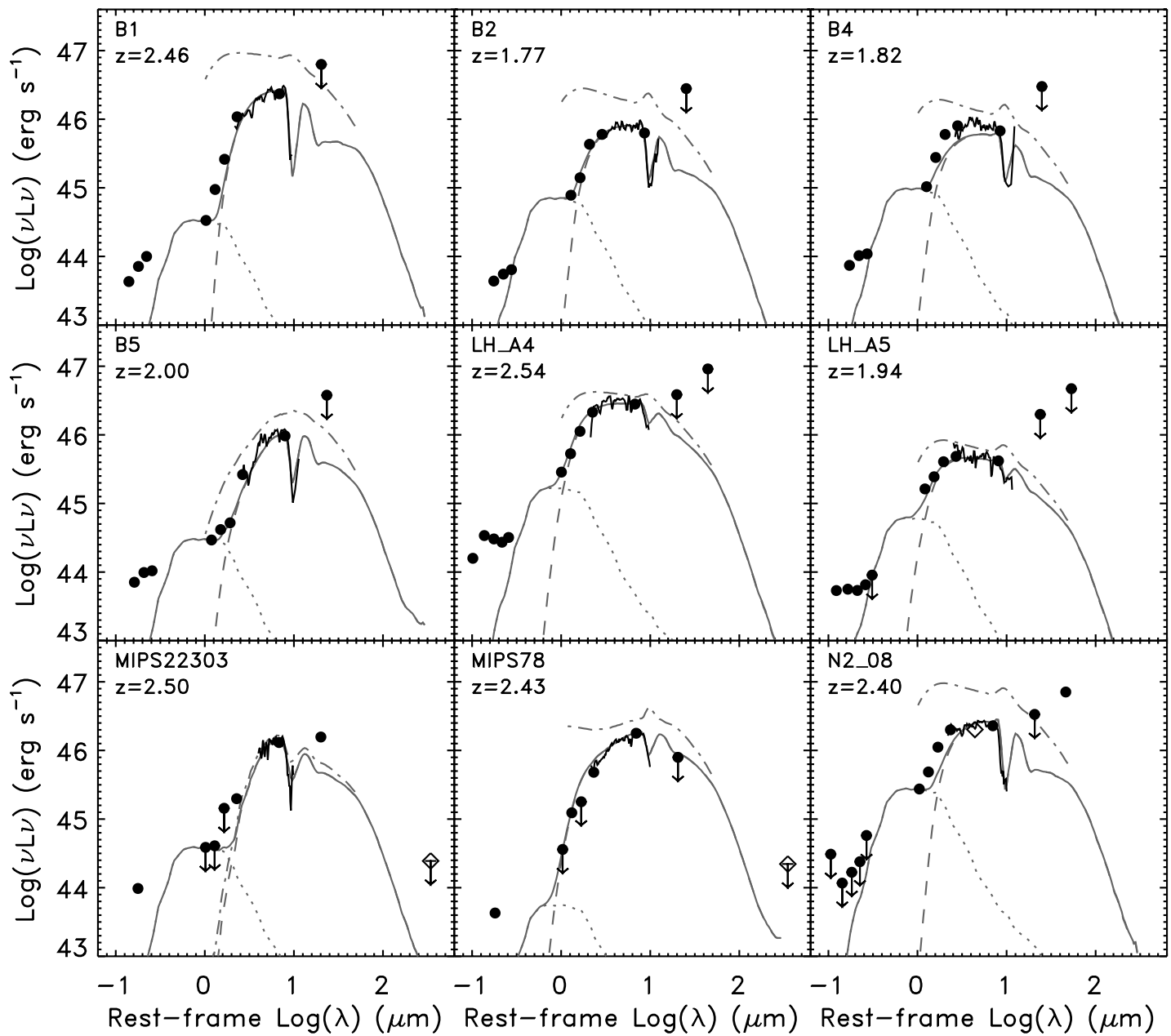

FIG. 3.- Rest-frame SEDs in $\nu L_{\nu}$ vs. $\lambda$ (black symbols and line) and best-fit model (solid gray line) obtained from a torus+cold absorber model (dashed gray line) and an elliptical template (dotted gray line) to fit the residuals in the NIR. The dot-dashed line represents the torus model, in the 1-50 $\mu$ m rest-frame wavelength range, before including the cold absorber. Symbols as in Fig. 1. The name and redshift of each source are annotated.

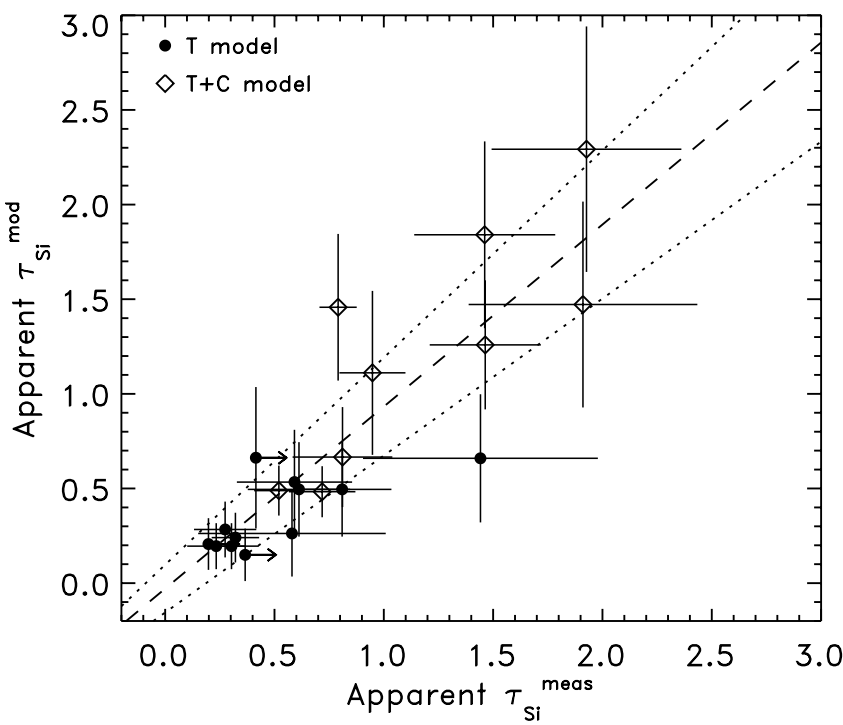

FIG. 4.-Comparison between the apparent Si optical depth at $9.7 \mu \mathrm{m}$ measured from the SED and spectrum, as described in $\S 2$, and from the torus models, as described in $\S 5$. Filled circles represent sources fitted with a T model; diamonds represent sources fitted with a $\mathrm{T}+\mathrm{C}$ model. The dashed line represents the best linear fit between the modeled and the measured $\tau_{\mathrm{Si}}$, and the dotted lines include the $1 \sigma$ uncertainties on the best-fit parameters. the FIR component (see values in Fig. 7). In those cases the bolometric luminosities are on average 2.5 times larger than estimated from the torus model. This implies a significant amount of cool dust that dominates the bolometric luminosity and that is located farther from the nucleus than the torus. This dust could be illuminated by radiation from the AGN that is not intercepted by the torus, which would be, qualitatively, consistent with the $\mathrm{T}+\mathrm{C}$ models described above. Overall, by taking into account the torus covering factor and an FIR component, the bolometric luminosities of our objects can be up to 7-17 times larger than estimated using equation (1).

\section{COMPOSITE IR SPECTRA}

In order to search for weak features in the IRS spectra of Figure 2, the individual spectra, after being boxcar smoothed to 3 times the approximate resolution of each module, were combined to obtain a composite spectrum with higher $\mathrm{S} / \mathrm{N}$. Composite spectra were made for two subsamples: (1) sources fitted with the T model (12 sources) and (2) sources fitted with the $\mathrm{T}+\mathrm{C}$ model (nine sources). The composite spectra were obtained by taking the median of all available data in the individual spectra in bins of $\Delta(\log \lambda)=0.005$ with $\lambda$ in $\mu \mathrm{m}$, after normalizing them at $6 \mu \mathrm{m}$ in the rest frame. The width of each bin is increased if less than three measurements are available, and it is reduced if more than 100 measurements are available. The uncertainty associated with 

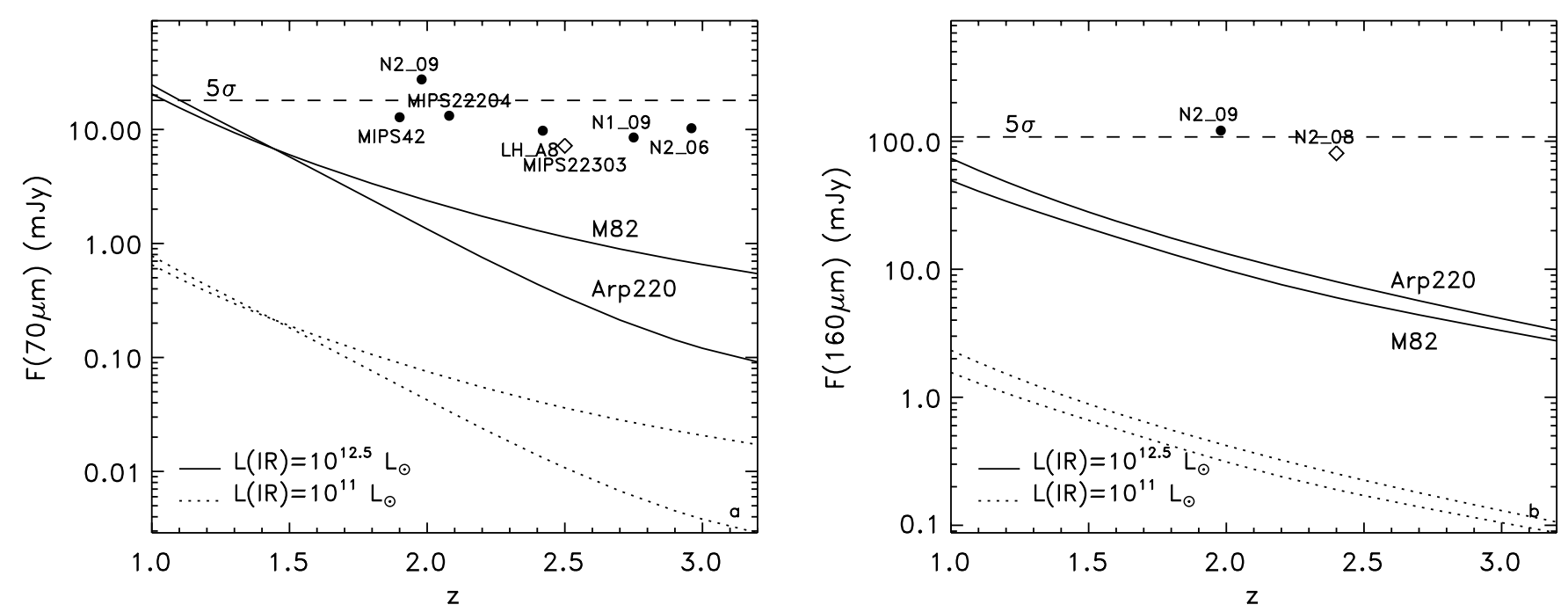

FIG. 5.-Predicted MIPS (a) $70 \mu \mathrm{m}$ and (b) $160 \mu \mathrm{m}$ fluxes for the starburst galaxies M 82 and Arp 220 with IR luminosities equal to $10^{11} L_{\odot}$ (dotted lines) and $10^{12.5} L_{\odot}$ (solid lines). The horizontal dashed line shows the SWIRE $5 \sigma$ limit. Black filled circles and diamonds represent sources in the selected sample detected at 70 or $160 \mu \mathrm{m}$ fitted with a $\mathrm{T}$ and a $\mathrm{T}+\mathrm{C}$ model, respectively. Source names are annotated.

the median value per bin corresponds to the uncertainty of the mean. The two composite spectra and associated $1 \sigma$ uncertainties are shown in Figure 8. The composite spectrum of the T group is characterized by a large dispersion, especially at $\lambda \simeq 10 \mu \mathrm{m}$. This large dispersion indicates that we combined sources with a broad range of depths in the Si feature.

Some spectral features other than Si that might be detected in the two composite spectra are highlighted, e.g., absorption features due to ice at $6.15 \mu \mathrm{m}$ and hydrocarbons (HACs) at $6.85 \mu \mathrm{m}$, the atomic emission features [Ar II] $6.99 \mu \mathrm{m},[\mathrm{Ne} \mathrm{VI}] 7.64 \mu \mathrm{m}$, and [S IV] $10.54 \mu \mathrm{m}$, the molecular hydrogen emission line $\mathrm{H}_{2}(\mathrm{~S} 3)$ 9.66 $\mu \mathrm{m}$, and the 6.2, 7.7, 8.6, and $11.3 \mu \mathrm{m}$ PAH emission features. Of these, the strongest feature that may be detected in both composite spectra is the $7.7 \mu \mathrm{m}$ PAH. This PAH is the strongest feature in starburst galaxies (Sturm et al. 2000; Brandl et al. 2006).

The strongest feature observed in the composite spectra is associated with molecular gas $\mathrm{H}_{2}, \mathrm{H}_{2}(\mathrm{~S} 3) 9.66 \mu \mathrm{m}$. The detection of such a feature cannot be confirmed because of the limited $\mathrm{S} / \mathrm{N}$ but would likely indicate the presence of shocked molecular hydrogen outside the MIR absorbing material, as can be expected in the case of high-velocity galaxy collisions, starburst superwinds, or AGN-driven outflows. Further observations would be necessary to test these scenarios.

Absorption due to HAC might be present in both composite spectra. This might be associated with the material that is also responsible for the deep Si feature, as in IRAS 00183-7111 (Spoon et al. 2004).

In order to facilitate a comparison between the two spectra, we overplotted a power-law model with slope of 2 normalized at $6 \mu \mathrm{m}$ in both panels of Figure 8. The main differences among the spectra are the 2-6 $\mu \mathrm{m}$ spectral slopes and the depths and shapes of the Si feature. In group 1 ( T model), the FWHM of the Si feature is $1.8 \mu \mathrm{m}$, the apparent optical depth is 0.4 , and the $2-6 \mu \mathrm{m}$ restframe power-law slope is 3.0 . In group $2(\mathrm{~T}+\mathrm{C}$ model $)$, the

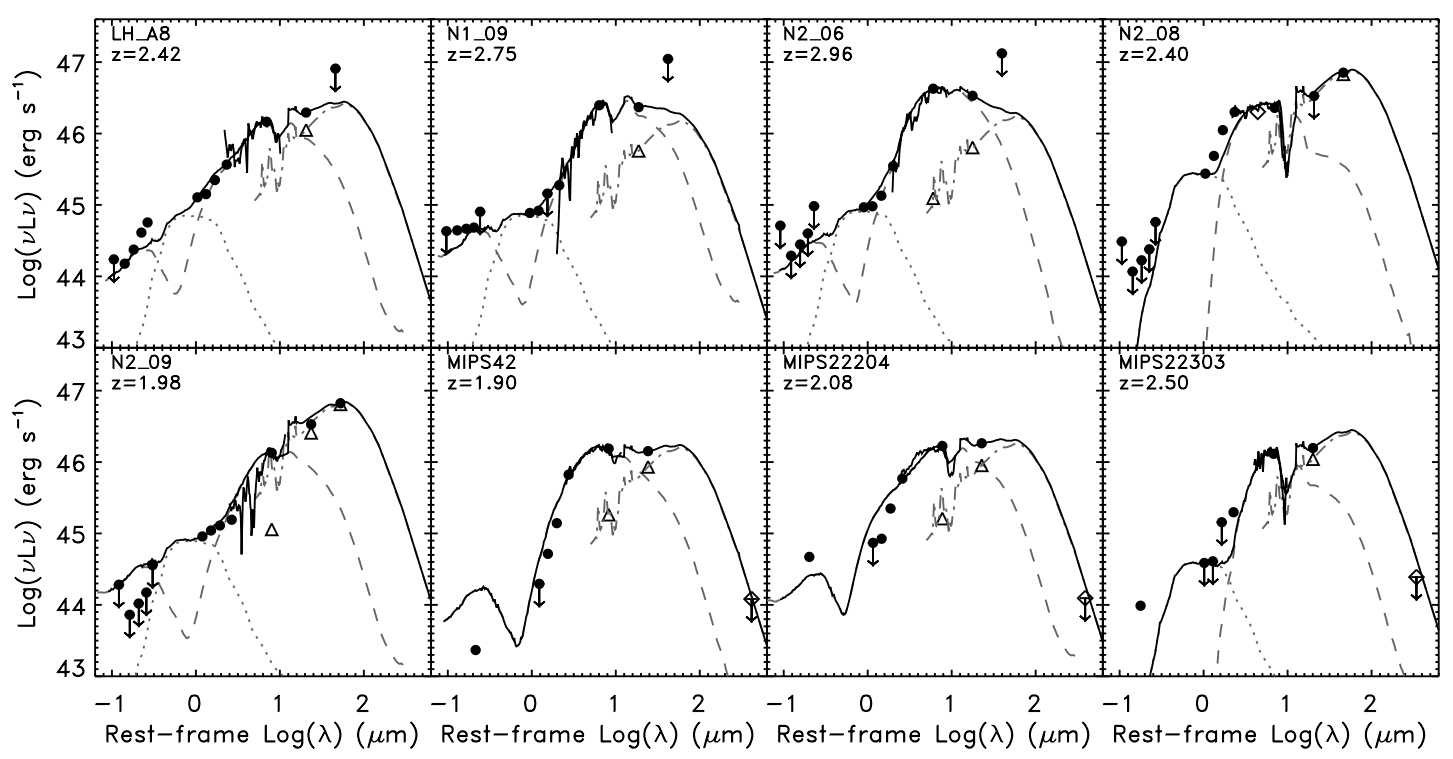

FIG. 6.- Rest-frame SEDs in $\nu L_{\nu}$ vs. $\lambda$ (black symbols and line) of sources detected at 70 or $160 \mu \mathrm{m}$. Also shown are the best-fit model (solid black line) obtained from a

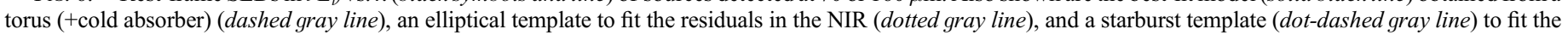
residuals in the FIR (triangles). Symbols as in Fig. 1. The name and redshift of each source are annotated. 


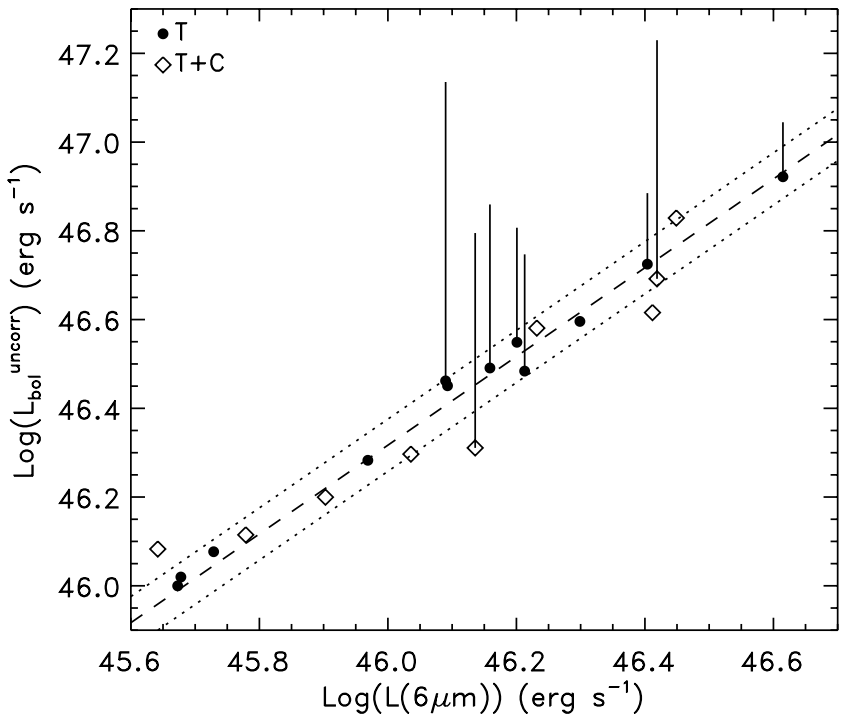

FIG. 7.-Comparison between the measured $6 \mu \mathrm{m}$ luminosity and the estimated AGN bolometric luminosity derived by integrating the best-fit torus model between 0.1 and $1000 \mu \mathrm{m}$. The dashed line corresponds to a linear fit with slope 1 , and the dotted lines correspond to the $1 \sigma$ dispersion. The bolometric luminosities derived by including the FIR luminosity in sources with FIR detections correspond to the top of the solid vertical lines. The additional FIR luminosity is derived by fitting the residuals in the FIR with an M82 template (see Fig. 6).

FWHM of the Si feature is $3.9 \mu \mathrm{m}$, the apparent optical depth is 0.9 , and the $2-6 \mu \mathrm{m}$ rest-frame power-law slope is 1.7 . It is interesting to note that, contrary to the expectation from torus models that the steepest NIR spectra are associated with the shallowest $\mathrm{Si}$ absorption feature, the spectrum with the deepest $\mathrm{Si}$ feature (group 2) shows instead the flattest NIR slope. This result supports the scenario proposed in $\S 5$ for the sources fitted with the $\mathrm{T}+\mathrm{C}$ models in which the hot dust close to the AGN is directly visible, and an additional absorber is required to reproduce the deep $\mathrm{Si}$ feature.

\section{COMPARISON WITH OTHER AGN SAMPLES}

\subsection{Fraction of Obscured QSOs}

In order to estimate the total surface density of the QSOs in our IRS sample, we first define empirically the colors that encompass our IRS sample. This is done by analyzing the location of our sources in various IR color-color diagrams. Then, we select a reference flux-limited sample of IR sources from a large and contiguous area that occupy the same location in the selected color-color diagrams. To select our reference sample, we used the SWIRE catalog in the Lockman Hole field.

It has been established that the majority of sources with red IRAC colors from Spitzer surveys have the colors expected from SEDs of AGNs at various redshifts (Lacy et al. 2004; Stern et al. 2005; Hatziminaoglou et al. 2005; Barmby et al. 2006). AGNs have such colors because of their hot dust emission. We thus analyzed several color-color diagrams combining the four IRAC bands and the MIPS $24 \mu \mathrm{m}$ band. From this analysis, we conclude that we can select sources with similar colors as our IRS sample by using only two color-color diagrams. One diagram combines all four IRAC bands, and it is shown in Figure $9 a$. The other diagram is made by combining three bands, IRAC[3.6], IRAC[8.0], and MIPS[24], and it is shown in Figure 9b. In both panels, we show our IRS sample and 1540 sources in a $1 \mathrm{deg}^{2}$ randomly selected field from the SWIRE survey. The 1540 sources are all detected in four IRAC bands and at $24 \mu \mathrm{m}$ to the SWIRE

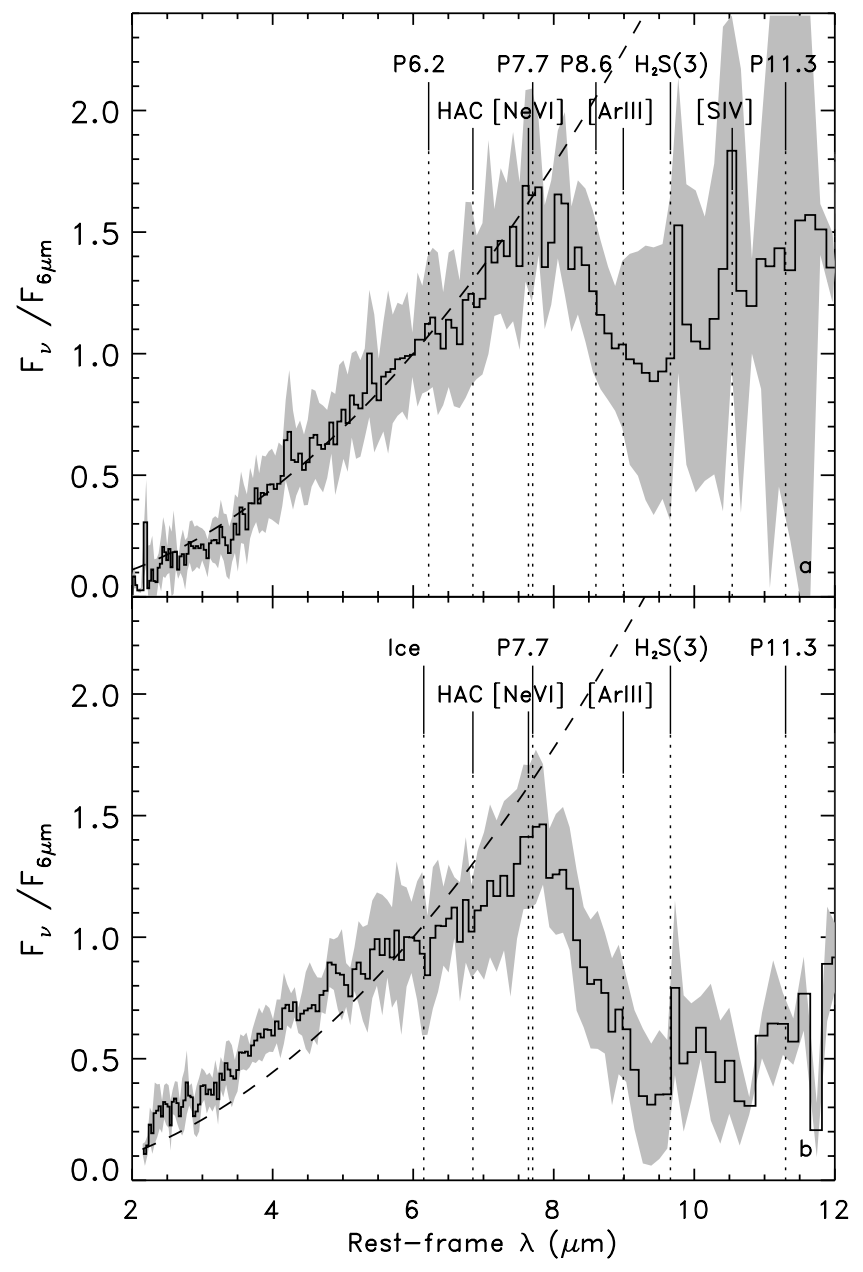

FIG. 8.- Median normalized IRS spectra of two subgroups of sources: (a) 12 sources modeled with the T model, and $(b)$ nine sources modeled with the $\mathrm{T}+\mathrm{C}$ model. The shaded area represents the mean absolute deviation. The dashed line represents a power law of slope $\alpha=2\left(F_{\nu} \propto \nu^{-\alpha}\right)$ normalized at $6 \mu \mathrm{m}$. Some spectral features that might be present are annotated.

limits in the Lockman Hole field (4.2, 7.5, 46.1, 47.3, and $209 \mu \mathrm{Jy}$ at 3.6, 4.5, 5.8, 8.0, and $24 \mu \mathrm{m}$, respectively).

The highly luminous and obscured QSOs in our IRS sample occupy the areas in the color-color diagrams with the reddest colors. The only template that shows similar colors corresponds to a highly obscured AGN (Polletta et al. 2006). Based on the areas in the diagrams occupied by our IRS sample, we define the following multicolor criteria to locate similar sources: $F(5.8) / F(3.6)>2$, $F(8.0) / F(4.5)>2$, and $\log [F(8.0) / F(3.6)]+\log [F(24) / F(3.6)]>$ 2.0. These color criteria are also shown in Figure 9 by dashed lines.

Having defined this color space for the highly luminous, obscured QSOs in our spectroscopic sample, we select from a random $3.5 \mathrm{deg}^{2}$ field within the SWIRE Lockman Hole field all sources detected in the four IRAC bands and with $24 \mu$ m fluxes greater than $1 \mathrm{mJy}$ with colors consistent with the color criteria mentioned above and shown in Figure 9.

Using all of these criteria, we find a total of 82 sources in $3.5 \mathrm{deg}^{2}$ in this random sample of SWIRE sources. To evaluate in more detail if all of these sources are similar to our obscured QSOs, we first visually inspected all of the available images to remove any source with spurious colors. We found one confused source, one saturated star, and two nearby galaxies with bad aperture photometric data. Thus, the sample was reduced from 82 to 78 sources $\left(22 \mathrm{deg}^{-2}\right)$. Next, we examined the overall SEDs of 

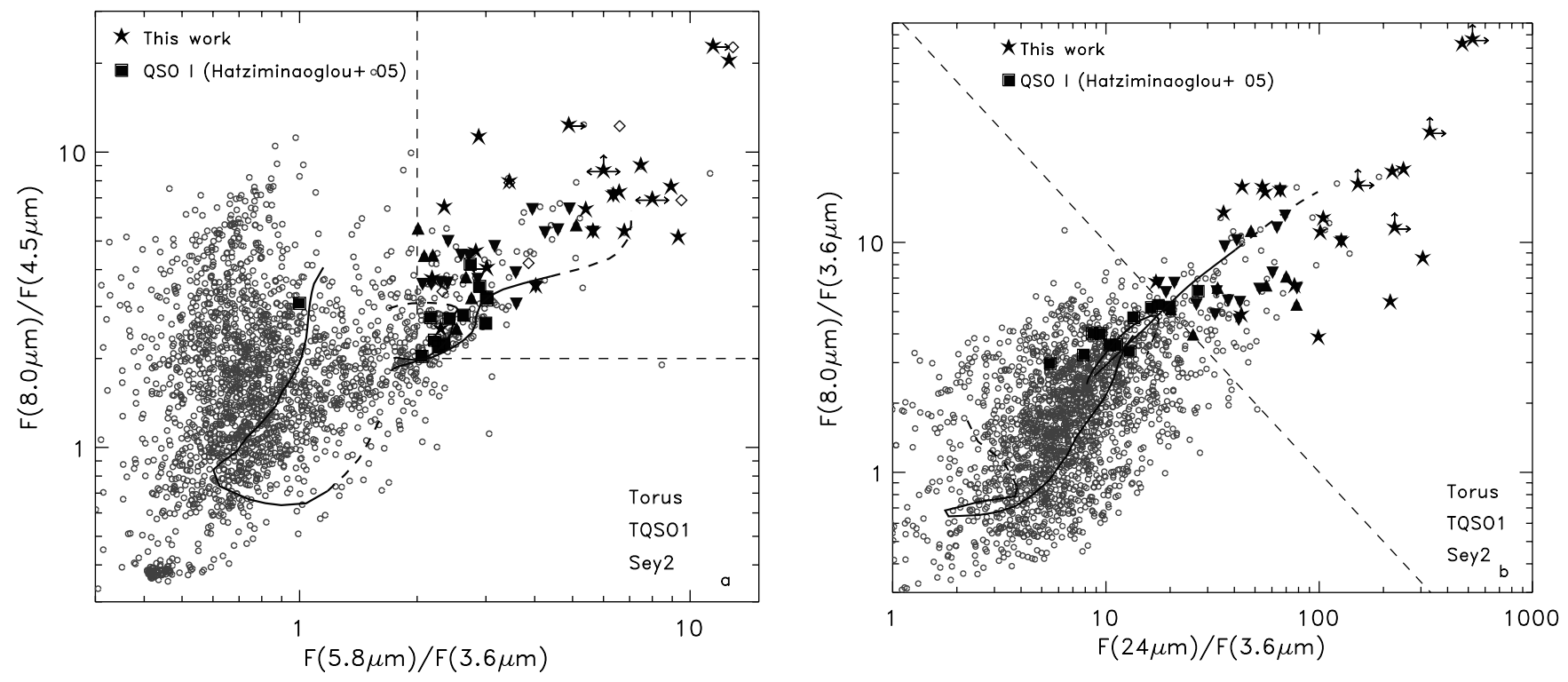

FIG. 9.- (a) IRAC color-color diagram. (b) IRAC-MIPS color-color diagram. The sources discussed in this paper are shown as stars. Circles represent all of the sources in a randomly selected $1 \mathrm{deg}^{2}$ field with $F(24 \mu \mathrm{m})>209 \mu \mathrm{Jy}$ and $F(8 \mu \mathrm{m})>47 \mu \mathrm{Jy}$. The filled squares represent the SDSS/SWIRE type 1 QSOs with $1<z<3$ from Hatziminaoglou et al. (2005). The lines show the color of templates of various types as annotated (solid: $0.1<z<2$; dashed: $2<z<3$ ). The regions to the right of the dashed lines represent the color criteria applied to select sources with colors consistent with those in our sample: unobscured QSO candidates (upward-pointing triangles) and obscured QSO candidates (downward-pointing triangles) (see $\S 7.1$ ). [See the electronic edition of the Journal for a color version of this figure.]

the 78 sources, including the optical data. Based on this analysis, we estimate that all selected sources have SEDs consistent with being AGN dominated and with those in our sample. We have previously found that sources classified as starbursts by IRS spectra can be distinguished from AGNs in the SWIRE samples based on their SEDs (Weedman et al. 2006a), so we can have confidence that the SED analysis does correctly locate AGNs.

To further test that our criteria select only AGN-dominated sources at $z=1.3-3.0$, we applied them to the large sample of 48 sources in Yan et al. (2007) for which an MIR classification, Spitzer IRAC and MIPS $24 \mu \mathrm{m}$ data, and spectroscopic redshifts are available. Our criteria select seven sources from this sample, of which four are included in our sample. The remaining three (MIPS 8034, MIPS 15958, and MIPS 22277) are all AGN dominated in the MIR with $\mathrm{EW}_{7.7 \mu \mathrm{m}}<0.22$; two fall into the redshift and luminosity range of our sample, but one (MIPS 8034) is at lower redshift $(z=0.95)$ and lower luminosity $[L(5.8 \mu \mathrm{m})=$ $\left.1.6 \times 10^{11} L_{\odot}\right]$. Note that the other two sources are not formally included in our IRS sample because MIPS 15958 has an MIR luminosity of $10^{11.95} L_{\odot}$, which is just below our selection threshold, and the IRS spectrum of MIPS 22277 shows some PAH emission. This test indicates that our sample is highly reliable in selecting AGNs, but it might include some ( $\frac{1}{7}$ or $14 \%$ ) sources at lower redshift and MIR luminosities.

We can perform an additional test on the degree of contamination from low-redshift and low-luminosity sources using the available optical spectroscopic data in the selected sample. Among the 78 selected sources, 12 sources have optical spectroscopic data. All 12 sources with spectroscopic data have redshifts from 1.3 to 3.0 , with a median value of 2.5 . This is the same range of redshifts found for our IRS sample, indicating that our selection succeeds in selecting sources in a similar redshift range. If one out of seven sources in our sample, or $14 \%$, were at lower $z$, as suggested by the analysis of the Yan et al. (2007) sample, we would have expected one to two low- $z$ sources in our spectroscopic sample. Since no low- $z$, low- $L$ interlopers were found in the spectroscopic sample, we can set a limit on the contamination fraction of $<8 \%$, which corresponds to 1 out of 12 sources, the minimum number we could have found in our spectroscopic sample. A redshift greater than 1.3 and the bright $24 \mu \mathrm{m}$ flux assure that also the MIR luminosity is high and consistent with those measured in our sample.

Since our selection of the 78 sources is only based on IR colors, it is possible that some of these sources are not as obscured as our IRS sample. We use the $F(3.6 \mu \mathrm{m}) / F$ (optical) flux ratio, where for the optical we used the three bands $g^{\prime}, r^{\prime}$, and $i^{\prime}$ to separate obscured and unobscured candidates. Based on previous studies (Polletta et al. 2006), we consider obscured candidates those with $F(3.6 \mu \mathrm{m}) / F\left(g^{\prime}, r^{\prime}, i^{\prime}\right)$ greater than 15,13 , and 10 , respectively, and unobscured candidates those with at least one flux ratio lower than those thresholds. This criterion yields 61 obscured AGN candidates and 17 unobscured AGN candidates. The optical-IR flux ratios of the QSO candidates are compared with those of our IRS sample and with other samples from the literature in Figure 10.

In order to verify our simple classification, we examined the optical spectral properties of the sources with available optical spectra and spectroscopic classification. A spectral classification is available for six sources, and they are all AGNs according to the observed emission lines; two show narrow emission lines and four show broad emission lines. Our classification agrees with the spectroscopic classification, in the sense that those with narrow lines are classified as obscured and those with broad lines as unobscured, in five out of six cases. The only exception is a source with Ly $\alpha, \mathrm{N} v \lambda 1240$, and $\mathrm{C}$ IV $\lambda 1549$ emission lines with FWHM $\sim 1700 \mathrm{~km} \mathrm{~s}^{-1}$, intermediate between a narrow and a broad emission line AGN. Although this comparison confirms our classification, we want to stress that our method based on IR/optical flux ratios is based only on a handful of sources whose availability of optical spectra is biased in favor of AGNs. Thus, any selection based on the criteria mentioned above should be applied with such a caveat in mind.

Based on this result alone, we would conclude that the surface density of obscured QSOs to the limits of the SWIRE survey is $17 \mathrm{deg}^{-2}$ (61 in $\left.3.5 \mathrm{deg}^{2}\right)$. However, there are a few sources with low $F(3.6 \mu \mathrm{m}) / F$ (optical) flux ratios, but with extremely red IR SEDs where the $3.6 \mu \mathrm{m}$ flux is much lower than the fluxes at 


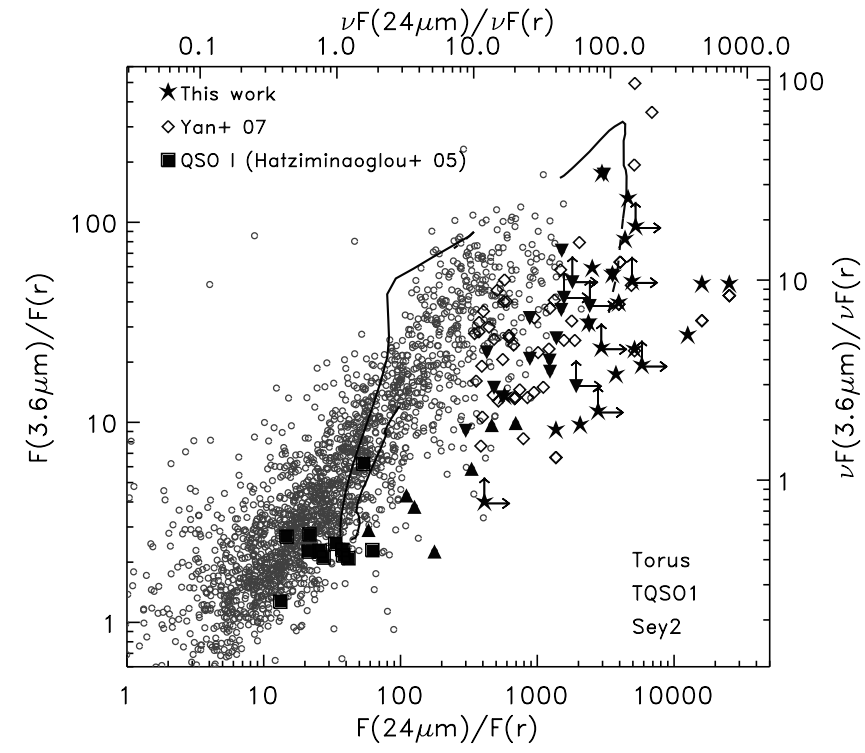

FIG. 10.-IR/optical flux ratios of the MIR-selected QSO sample (stars) and of the SWIRE sources with similar IR colors (triangles) (selected as shown in Fig. 9). The $F(3.6 \mu \mathrm{m}) / F\left(r^{\prime}\right)$ flux ratio shows one of the criteria applied to separate obscured (downward-pointing triangles) and unobscured (upward-pointing triangles) QSO candidates. Symbols as in Fig. 9. [See the electronic edition of the Journal for a color version of this figure.]

longer wavelengths. Thus, it is likely that some unobscured QSO candidates might actually be obscured or reddened type 1 AGNs. In such a case, we might be underestimating the surface density of obscured QSOs.

Indeed, our color selection criteria rule out most of the type 1 QSOs such as those in Hatziminaoglou et al. (2005). The IR colors for the SDSS/SWIRE type 1 QSOs in the 1.3-3.0 redshift range are also shown for comparison in Figure 9. While there is a large overlap between the SDSS/SWIRE type 1 QSO sample and our obscured sample in IRAC colors (Fig. 9a), the SDSS/SWIRE type 1 QSOs are all clustered toward the bluest side of the color distribution. Moreover, there is almost a complete separation in the $F(24) / F(3.6)$ color between the two samples, with all the SDSS/SWIRE type 1 QSOs having $F(24) / F(3.6)<30$, and all but one of the obscured QSOs having $F(24) / F(3.6)>30$. This difference is likely due to the large NIR extinction affecting our sample, which is absent in the SDSS/SWIRE type 1 QSO sample and highlights the ability of this flux ratio to distinguish obscured from unobscured QSOs. Thus, our color selection criteria disfavor the classical type 1 QSOs. If we consider all selected sources as obscured AGN candidates, their surface density increases to $22 \mathrm{deg}^{-2}$ (78 in $3.5 \mathrm{deg}^{2}$ ).

In order to derive the fraction of obscured QSOs among the $24 \mu \mathrm{m}$ bright AGN population at $z=1.3-3.0$ and with MIR luminosities $>10^{12} L_{\odot}$, we need to estimate the surface density of unobscured, type 1 QSOs with the same redshift range and MIR luminosities as our IRS sample. We estimate such a surface density from the $8 \mu \mathrm{m}$ luminosity function derived by Brown et al. (2006) for all AGNs with $F(24 \mu \mathrm{m})>1 \mathrm{mJy}$. Assuming the same QSO template as in Brown et al. (2006), the derived $8 \mu \mathrm{m}$ absolute magnitude corresponding to $L(6 \mu \mathrm{m})=10^{12} L_{\odot}$ is $M_{8} \mu \mathrm{m}=$ -28.39 . The estimated surface density of all type 1 AGNs with $M_{8 \mu \mathrm{m}}<-28.39$ in the redshift range $1.3<z<3.0$ is $11.7 \mathrm{deg}^{-2}$. A value only slightly smaller, $9.4 \mathrm{deg}^{-2}$, is derived assuming the optical luminosity function for type 1 AGNs measured by Richards et al. (2006) and $M_{B}<-22.3$, which corresponds to $M_{8 \mu \mathrm{m}}<$ -28.39 assuming the same template. Using the result derived

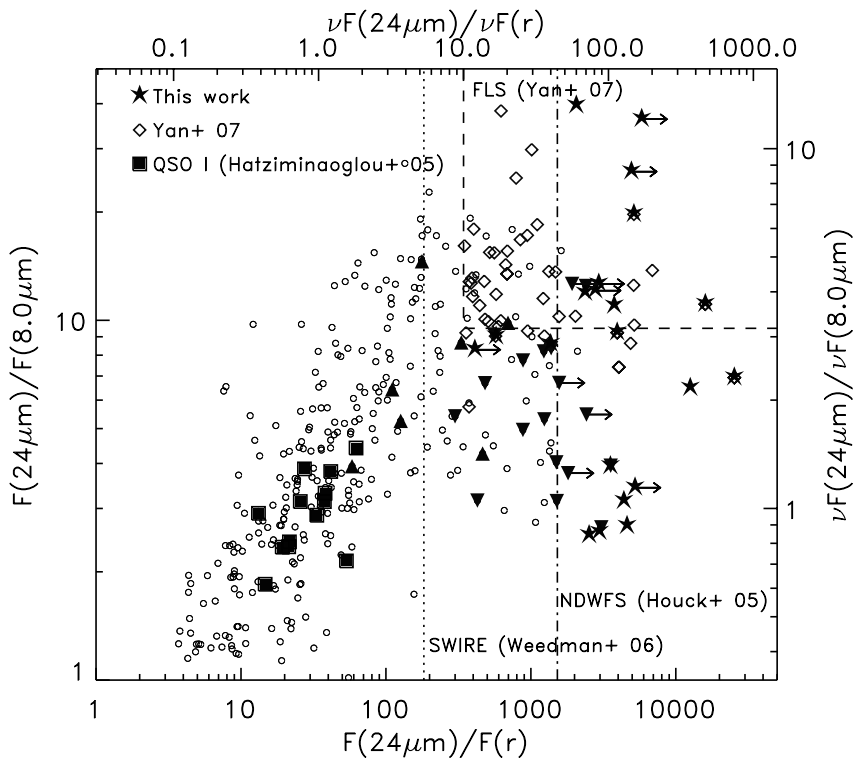

FIG. 11.-Color-color diagram showing the selection of the MIR-selected samples from which some of the sources in this work are drawn. The dotted line shows $F(24 \mu \mathrm{m})>0.9 \mathrm{mJy}$ and $\operatorname{mag}\left(r^{\prime}\right)>22$ from Weedman et al. (2006a) (note that they also require an X-ray detection). The dot-dashed line shows $F(24 \mu \mathrm{m})>$ $0.75 \mathrm{mJy}$ and $\operatorname{mag}(R)>24.5$ from Houck et al. (2005). The region enclosed by the dashed lines shows $\log [\nu F(24 \mu \mathrm{m}) / \nu F(8 \mu \mathrm{m})] \geq 0.5$ and $\log [\nu F(24 \mu \mathrm{m}) /$ $\left.\nu F\left(r^{\prime}\right)\right] \geq 1.0$ from Yan et al. (2007). For comparison we show all the SWIRE sources with $F(24 \mu \mathrm{m})>1 \mathrm{mJy}$ in $1 \mathrm{deg}^{2}$ (open circles), the SDSS/SWIRE type 1 QSO from Hatziminaoglou et al. (2005) (squares), the MIR-selected sources in Yan et al. (2007) (diamonds), and the SWIRE sources detected at 8 and $24 \mu \mathrm{m}$ selected as shown in Fig. 9 (downward-pointing triangles and upward-pointing triangles). Symbols as in Fig. 9. [See the electronic edition of the Journal for a color version of this figure.]

from the MIR-selected QSO sample, the ratio of obscured to unobscured MIR-luminous QSOs in the 1.3-3.0 redshift range is, therefore, $(17-22) / 11.7$, or $\sim 1.5-1.9$. This value is consistent with the fraction of obscured AGNs measured in MIR-radioselected samples (Martínez-Sansigre et al. 2005).

\subsection{Comparison with Other IRS MIR-selected Samples}

As described in $\S 2$, the main selection criteria applied to carry out IRS observations of Spitzer sources were bright $24 \mu \mathrm{m}$ fluxes and faint optical magnitudes (Houck et al. 2005). In addition, other selection criteria were applied in some cases to favor the selection of starburst or AGN systems. In order to favor starburstdominated galaxies, Yan et al. (2007) also require large $F(24 \mu \mathrm{m}) /$ $F(8 \mu \mathrm{m})$ flux ratios. Weedman et al. (2006a) require an X-ray detection to select AGNs and a bump in the IRAC SED to select starbursts.

In Figure 11 we compare the $F(24 \mu \mathrm{m}) / F(8 \mu \mathrm{m})$ and $F(24 \mu \mathrm{m}) /$ $F\left(r^{\prime}\right)$ flux ratios of the sources in our IRS sample with the selection criteria applied to select the sample from which a large fraction of our sources were drawn (Houck et al. 2005; Yan et al. 2007; Weedman et al. 2006a). We also show for comparison the colors of the sources in Yan et al. (2007), of those selected by the IR criteria described in the previous section, of the SDSS/SWIRE type 1 AGNs from Hatziminaoglou et al. (2005), and of a random sample of SWIRE sources with $F(24 \mu \mathrm{m})>1 \mathrm{mJy}$ in $1 \mathrm{deg}^{2}$.

It is interesting to note the separation in $F(24 \mu \mathrm{m}) / F(8 \mu \mathrm{m})$ flux ratios between the selected sample and the starburst and composite sample from Yan et al. (2007). Lower $F(24 \mu \mathrm{m}) / F(8 \mu \mathrm{m})$ flux ratios favor AGN-dominated sources, but high ratios do not exclusively select starburst galaxies. Indeed, half of our sources show large $F(24 \mu \mathrm{m}) / F(8 \mu \mathrm{m})$, and a large fraction of sources in 

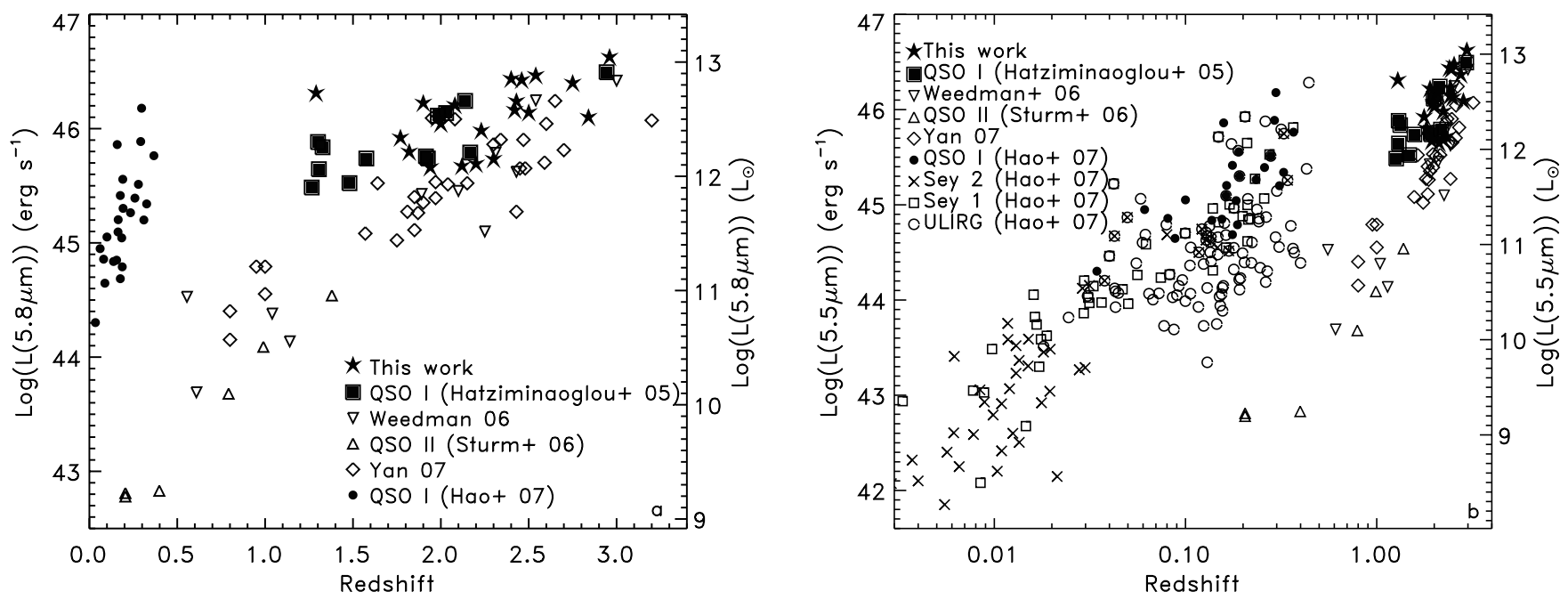

FIG. 12.- (a) MIR ( $6 \mu \mathrm{m}$ rest frame) luminosities vs. redshift for the obscured AGNs in this work ( filled stars) and various AGN samples: X-ray-selected type 2 QSOs (upward-pointing triangles; Sturm et al. 2006), X-ray-selected optically faint AGNs (downward-pointing triangles; Weedman et al. 2006a), IR-selected AGNs and composite sources (diamonds; Yan et al. 2007), SDSS/SWIRE-selected type 1 QSOs (squares; Hatziminaoglou et al. 2005), and type 1 QSOs ( filled circles; Hao et al. 2007). (b) Same data as shown in (a), but with, in addition, the values for the Seyfert 1 galaxies (squares), Seyfert 2 galaxies (crosses), and ULIRGs (open circles) from Hao et al. (2007). [See the electronic edition of the Journal for a color version of this figure.]

Yan et al. (2007) contain an important AGN component (Sajina et al. 2007a).

Our sample shows the largest $F(24 \mu \mathrm{m}) / F\left(r^{\prime}\right)$ ratios of all samples. The sources selected in the previous section to derive the surface density of our IRS sample show $F(24 \mu \mathrm{m}) / F\left(r^{\prime}\right)$ flux ratios in between the SDSS/SWIRE type 1 QSOs and the IRS sources. This indicates that the sources presented here might actually be more extreme than the sample selected above.

\subsection{Luminosity, $\tau_{\mathrm{Si}}$, and Redshift}

The MIR $(6 \mu \mathrm{m})$ luminosities and redshifts of the AGN sample presented here are compared with those of other AGN samples from the literature in Figure 12. The literature samples include X-ray-selected type 2 QSOs (Sturm et al. 2006), optically and IR-selected (SDSS/SWIRE) type 1 QSOs with $1<z<3$ (Hatziminaoglou et al. 2005), X-ray-selected, optically faint $(r>22)$ and IR-bright $[F(24 \mu \mathrm{m})>1 \mathrm{mJy}]$ sources (Weedman et al. 2006a), MIR-selected AGNs and composite sources (Yan et al. 2007), and various types of local AGNs, type 1 QSOs, Seyfert 1 and Seyfert 2 galaxies, and ULIRGs (Hao et al. 2007). Note that some of the sources in our sample are in common with those in Weedman et al. (2006a; five sources) and with those in Yan et al. (2007; five sources).

Our sources are among the most luminous MIR sources in this compilation. The only sources with similar MIR luminosities are the SDSS/SWIRE type 1 QSOs from Hatziminaoglou et al. (2005) and the most luminous type 1 QSOs from Hao et al. (2007). The composite sources in Yan et al. (2007) and the type 2 QSOs from Sturm et al. (2006) are on average 3 and 250 times fainter, respectively.

The MIR $(5.5 \mu \mathrm{m})$ luminosities and the measured apparent $\mathrm{Si}$ optical depth, $\tau_{\mathrm{Si}}$, of our AGN sample are compared with those of other AGN samples from some of the samples in the above compilation in Figure 13. The literature samples include all AGNs in Hao et al. (2007): type 1 QSOs, ULIRGs, and Seyfert 1 and Seyfert 2 galaxies. Our sample covers a new parameter space in the MIR luminosity-absorption distribution of all known AGNs, as illustrated in Figure 13. The selected obscured QSOs show the highest MIR luminosities $\left[L(5.5 \mu \mathrm{m})>10^{12} L_{\odot}\right]$, consistent only with the most luminous type 1 QSOs and Seyfert 1 galaxies, and also show large Si optical depths, up to $\tau_{\mathrm{Si}}=3.2$, consistent with those observed in ULIRGs and in some Seyfert 2 galaxies.

\section{X-RAY PROPERTIES}

X-ray data from Chandra are available for 11 sources, but only five are detected (Weedman et al. 2006a; Polletta et al. 2006; LH_02, LH_A4, LH_A5, LH_A6, and LH_A8). Chandra $5 \mathrm{ks}$ observations are available for the five sources in the Bootes field (Murray et al. 2005), but none of the selected sources are detected. We report an upper limit to the broadband $(0.3-8 \mathrm{keV})$ X-ray flux for these sources corresponding to their $90 \%$ completeness limit $\left(10^{-14} \mathrm{ergs} \mathrm{cm}^{-2} \mathrm{~s}^{-1}\right.$; Murray et al. 2005). LH_A11 is part of the $70 \mathrm{ks}$ SWIRE/Chandra survey (Polletta et al. 2006)

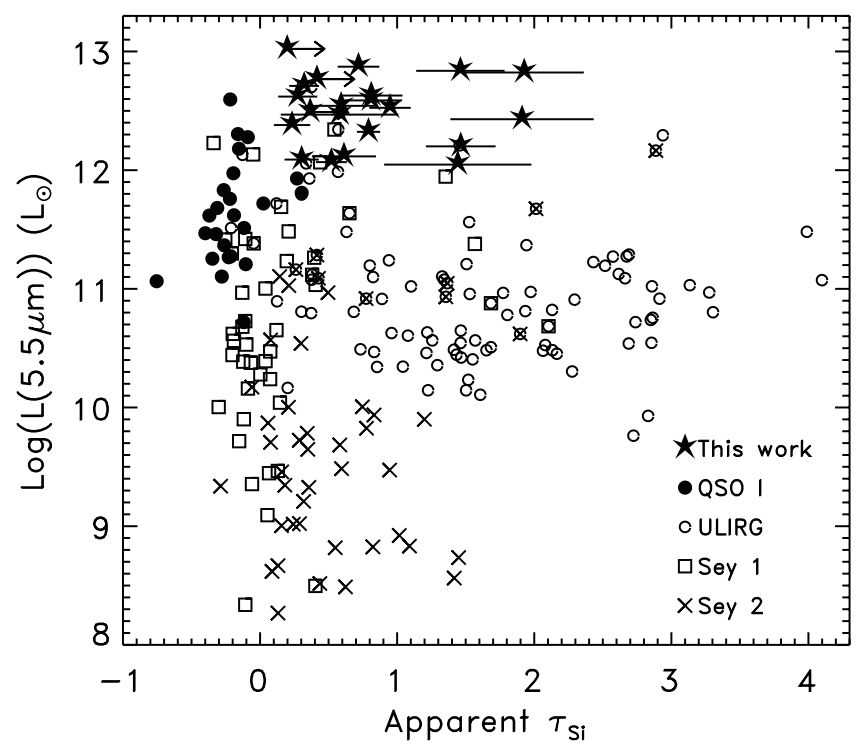

FIG. 13.- MIR (5.55 $\mu \mathrm{m}$ rest frame) luminosities vs. the Si apparent optical depth at $9.7 \mu \mathrm{m}$ of the obscured AGNs in this work ( filled stars) and of various AGN samples from Hao et al. (2007): type 1 QSOs ( filled circles), ULIRGs (open circles), Seyfert 1 galaxies (open squares), and Seyfert 2 galaxies (crosses). Rightward-pointing arrows represent lower limits to $\tau_{\mathrm{Si}}$. [See the electronic edition of the Journal for a color version of this figure.] 
TABLE 5

X-Ray Properties

\begin{tabular}{|c|c|c|c|c|}
\hline Source ID & $\begin{array}{c}F_{0.3-8 \mathrm{keV}^{\mathrm{a}}} \\
\left(10^{-15} \mathrm{ergs} \mathrm{cm}^{-2} \mathrm{~s}^{-1}\right)\end{array}$ & $\begin{array}{c}N_{\mathrm{H}}^{\text {eff } \mathrm{b}} \\
\left(10^{22} \mathrm{~cm}^{-2}\right)\end{array}$ & $\begin{array}{l}\log L_{0.3-8} \mathrm{keV} \\
\left(\mathrm{ergs} \mathrm{s}^{-1}\right)\end{array}$ & $\begin{array}{l}\log L_{0.3-8 \mathrm{keV}}^{\text {corr }}{ }^{\mathrm{c}} \\
\left(\mathrm{ergs} \mathrm{s}^{-1}\right)\end{array}$ \\
\hline 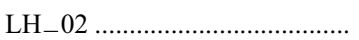 & 4.51 & $0.12_{-0.00}^{+4.10}$ & 44.07 & 44.08 \\
\hline LH_A4 & 1.95 & $105_{-29}^{+192}$ & 43.83 & 45.08 \\
\hline LH_A5 & 19.31 & $9.4_{-3.1}^{+4.3}$ & 44.61 & 45.06 \\
\hline 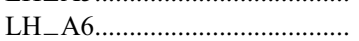 & 24.94 & $6.3_{-24}^{+3.5}$ & 44.78 & 45.10 \\
\hline LH_A8 & 4.45 & $40_{-17}^{+29.4}$ & 44.19 & 45.00 \\
\hline 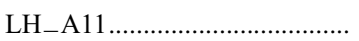 & $<1.0$ & $\ldots$ & $<43.46$ & $\ldots$ \\
\hline В1 & $<10.0$ & $\ldots$ & $<44.53$ & $\ldots$ \\
\hline 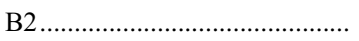 & $<10.0$ & $\ldots$ & $<44.21$ & $\ldots$ \\
\hline 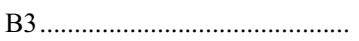 & $<10.0$ & $\ldots$ & $<43.89$ & $\ldots$ \\
\hline 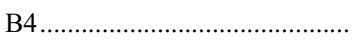 & $<10.0$ & $\ldots$ & $<44.23$ & $\ldots$ \\
\hline В5 & $<10.0$ & $\ldots$ & $<44.33$ & $\ldots$ \\
\hline
\end{tabular}

Noтes.-For more details on the X-ray data see Polletta et al. (2006). Upper limits to the X-ray fluxes correspond to $90 \%$ completeness.

a Broadband $(0.3-8 \mathrm{keV}) \mathrm{X}$-ray flux.

${ }^{\mathrm{b}}$ Effective column density derived from the hardness ratios assuming that the intrinsic spectrum is a power-law model with photon index $\Gamma$ equal to 1.7 and Galactic $N_{\mathrm{H}}\left(6 \times 10^{19} \mathrm{~cm}^{-2}\right)$. Uncertainties reflect only the statistical errors from the observed counts and do not include uncertainties in the spectral model.

${ }^{c}$ Absorption-corrected X-ray luminosities derived assuming the effective column density, $N_{\mathrm{H}}^{\mathrm{eff}}$, and an intrinsic spectrum modeled with a power-law model with photon index $\Gamma=1.7$.

but was not detected, thus we report an upper limit to the broadband flux of $10^{-15} \mathrm{ergs} \mathrm{cm}^{-2} \mathrm{~s}^{-1}$. Chandra observations for B2 are scheduled (CXO proposal 08700396) and XMM-Newton observations are pending for N2_06, B1, N2_08, and B3 (XMMNewton proposal 050326). In Table 5 we list observed X-ray fluxes, estimated effective column densities and luminosities for the detected five sources, and upper limits to the fluxes for the remaining six sources. Here we compare the absorption measured in the $\mathrm{X}$-rays with the extinction measured in the IR and use the MIRto-X-ray luminosity ratio to investigate the dust covering fraction in the selected sample.

\subsection{X-Ray Absorption and IR Extinction}

The effective gas column densities responsible for absorption in the $\mathrm{X}$-rays are derived from the $\mathrm{X}$-ray hardness ratios using the method described in Polletta et al. (2006). The estimated values are reported in Table 5. The absorption measured in the X-rays and the dust extinction measured in the IR, $\tau_{\mathrm{Si}}$, are compared in Figure 14. We also show a curve (dotted line) corresponding to equivalent $N_{\mathrm{H}}$ and $\tau_{\mathrm{Si}}$ assuming a gas-to-dust ratio of 100 , instead of the Galactic value, a dust density of $2.5 \mathrm{~g} \mathrm{~cm}^{-3}$, and 0.1 $\mu \mathrm{m}$ radius per dust grain, or $N_{\mathrm{H}} \simeq 2.26 \times 10^{22} \tau_{\mathrm{Si}} \mathrm{cm}^{-2}$.

In four sources, the $N_{\mathrm{H}}$ is significantly larger than that derived in the IR. The only exception, where the $N_{\mathrm{H}}$ is lower, is LH_A2, which is characterized by a soft X-ray spectrum. However, there is evidence, based on the X-ray/IR luminosity ratio, that in LH_A2 the observed X-ray spectrum corresponds to a small fraction of the intrinsic X-ray emission due to scattering, while the intrinsic emission is absorbed by a Compton-thick column density $\left(N_{\mathrm{H}}>\right.$ $10^{24} \mathrm{~cm}^{-2}$ ). This source is also characterized by the highest IR extinction.

The large $N_{\mathrm{H}}$ values compared to the extinction measured in the IR by $\tau_{\mathrm{Si}}$ are easily explained. The apparent $\mathrm{Si}$ optical depth underestimates the true extinction in the IR because its measurement does not take into account reemission of radiation at the same wavelengths where absorption takes place. Moreover, we expect the correlation between the two measurements of absorption to have a wide dispersion because the absorption measured in the X-rays can be highly variable (see, e.g., Risaliti et al. 2007) and because the two columns, in the X-ray and in the IR, are mea- sured along different paths as the emitting sources have different spatial locations and distributions. It is also quite plausible that, in addition to the absorption produced by the medium that is responsible for the extinction seen in the IR, the X-ray source suffers absorption by gas clouds, possibly ionized, close to the nucleus that may be dust-free and thus do not contribute to any emission or absorption in the IR.

A similar comparison was presented by Shi et al. (2006) for a large compilation of AGNs of different type. Instead of the apparent Si optical depth, $\tau_{\mathrm{Si}}$, they define the Si strength as an indicator of IR extinction. The Si strength is equivalent to $e^{-\tau_{\mathrm{Si}}}-1$; thus, for our sources it ranges from -0.3 to -0.8 . We report their best linear fit obtained for their entire AGN sample in Figure 14 after converting the Si strength to the apparent $\tau_{\mathrm{Si}}$. By comparing our sources with their relationship and their Figure 3, we find that our sources occupy the lower boundary of the $\mathrm{Si}$ strength $-N_{\mathrm{H}}$ relation they observe and overlap with the most IR-obscured sources in their sample, which are all Seyfert 2 galaxies. Thus, the $N_{\mathrm{H}}$ and $\tau_{\mathrm{Si}}$ of our sources are consistent with those found in other AGNs, but they overlap with those observed in the most IR-obscured ones. This is a consequence of our sample selection.

\subsection{Dust Covering Fraction}

The dust covering fraction can be estimated by comparing the thermal reprocessed emission, e.g., $L(6 \mu \mathrm{m})$, and the luminosity of the heating source. The best proxy for the intensity of the heating source is given by the ultraviolet (UV) luminosity, since UV photons are more efficiently thermalized than those at other wavelengths (both X-ray and IR). Since we do not know the intrinsic (before absorption) UV luminosity because it is mostly absorbed and only few sources are detected in the optical, we adopt the absorption-corrected X-ray luminosity as a proxy for the intensity of the heating source. Since the ratio between the UV and X-ray luminosity varies with luminosity (e.g., Steffen et al. 2006), in the sense that more luminous AGNs have lower X-ray/UV luminosity ratios, any trend of this ratio with the X-ray luminosity must be carefully interpreted. With this caveat in mind, we adopt the ratio between $L(6 \mu \mathrm{m})$ and $L_{0.3-8 \mathrm{keV}}^{\text {corr }}$ as an approximate indicator of dust covering fraction. 


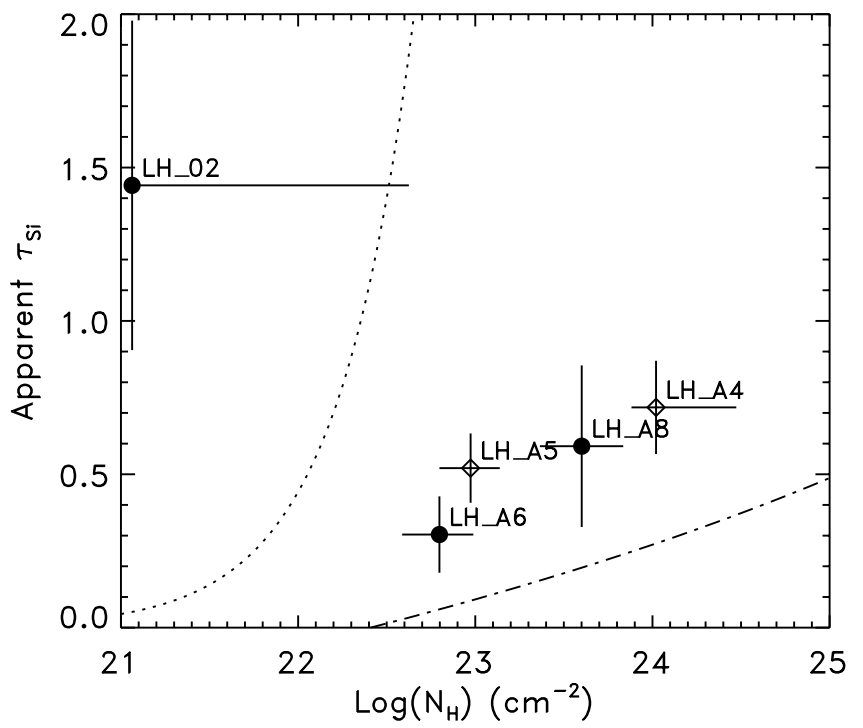

FIG. 14.-Comparison between the apparent Si optical depth and the column density estimated from the X-ray hardness ratio. The dotted line corresponds to the expected agreement in case both absorptions occur in the same medium and assuming a Galactic gas-to-dust ratio of 100 . The dot-dashed line corresponds to the linear fit found for a large compilation of AGNs by Shi et al. (2006).

In Figure 15 we show the MIR/X-ray luminosity ratios of our sample. In the case of non-X-ray detections, we show the X-ray luminosity corresponding to the upper limit to the flux. Since the flux is lower than the assumed upper limit, the intrinsic luminosity could be much lower than estimated, but since we do not correct for absorption, it can also be higher than estimated. The luminosity ratios for these sources are thus reported with an uncertainty of a factor of 10 .

We also report for comparison the luminosity ratios typical of AGNs (region enclosed by the dashed lines in Fig. 15) and of the X-ray-selected type 2 QSOs from Sturm et al. (2006) (hereafter the S06 sample). The range of ratios for AGNs was obtained from a compilation of ratios for various types of AGNs (adapted from Polletta et al. 2007). These authors find that the observed range of MIR-to-X-ray luminosity ratios in AGNs is characterized by a wide dispersion, from 0.3 to 13 , with no dependency on AGN type and luminosity, confirming previous results (see e.g., Lutz et al. 2004; Sturm et al. 2005, 2006; Horst et al. 2006). The sources in the S06 sample are heavily absorbed in the X-rays, and their MIR emission is mostly due to the AGN, but they do not show the Si feature in absorption. The ratios for the S06 sample are consistent with those typical of AGNs.

Interestingly, most ( 9 out of 11) of the sources in our sample show higher ratios than the literature samples and the S06 sample. The observed offset is not due to a different range of X-ray luminosities, as it is observed across the entire luminosity range of our sample. Yet, it is difficult to establish whether there is a clear difference among our sample and the others. For example, the X-ray luminosity of LH_02 is likely underestimated. Indeed, its soft X-ray spectrum and low X-ray luminosity suggest that its observed X-ray emission is scattered light and the intrinsic luminosity is completely absorbed. Moreover, the uncertainties associated with the absorption-corrected X-ray luminosities can be quite large since these are typically faint sources and the column densities are derived from the hardness ratios rather than from X-ray spectral modeling. Deeper X-ray data for these sources or X-ray observations of similar depth as those already available for the rest of the sample would be necessary to confirm such a result. If confirmed, this offset in $L_{\mathrm{MIR}} / L_{\mathrm{X}}$ might be simply due to

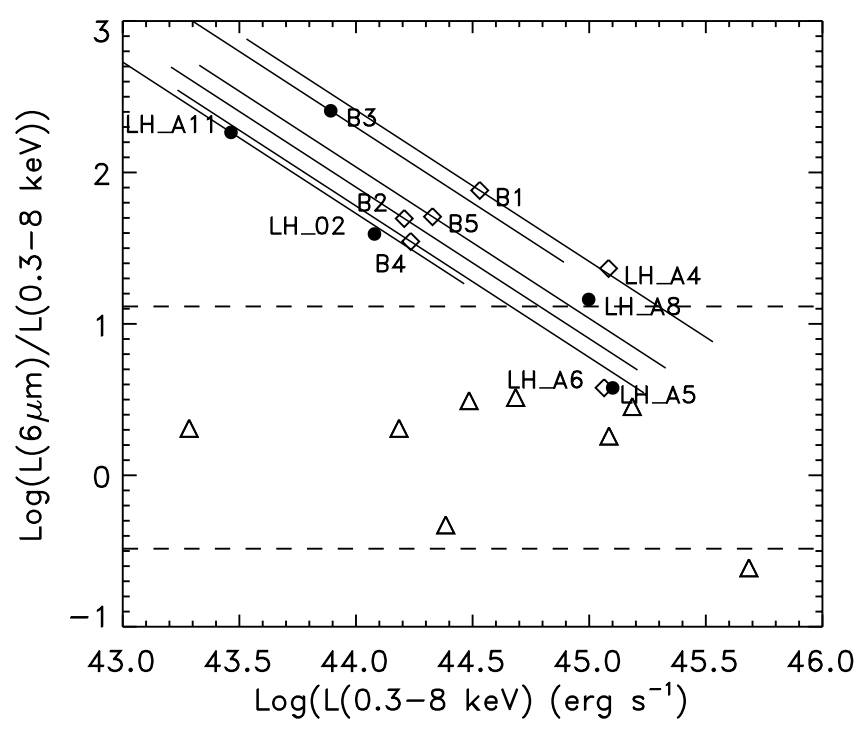

FIG. 15.-MIR (6 $\mu \mathrm{m}$ rest frame) over X-ray luminosity as a function of X-ray luminosity for the sources presented in this work ( filled circles and open diamonds) and the type 2 QSOs in Sturm et al. (2006; triangles). Filled circles are sources fitted with the T model, and open diamonds are sources fitted with the $\mathrm{T}+\mathrm{C}$ model. The $\mathrm{X}$-ray luminosities have been corrected for absorption. Large error bars on the luminosity ratio (a factor of 10 in the X-ray luminosity) are reported for sources for which only an upper limit to the X-ray flux is available. The dashed lines enclose the region where the majority of AGNs lie (adapted from Polletta et al. 2007).

a selection bias in our sample. A bias could have been introduced by the powerful AGN emission at MIR wavelengths, which might be an indicator for a specific dust geometry, e.g., a more compact cloud distribution in the vicinity of the nucleus. Also the presence of $\mathrm{Si}$ absorption might have introduced a bias in favor of dustier sources and thus with larger dust covering factors. However, we do not expect a correlation between deep Si features in absorption and covering factors, i.e., large $L(\mathrm{MIR}) / L$ (X-ray) luminosity ratios, if the medium producing $\mathrm{Si}$ absorption is clumpy as suggested by Nenkova et al. (2002), Dullemond \& van Bemmel (2005), Hönig et al. (2006), and Shi et al. (2006). Indeed, the LOS might not intercept any cloud, even if the covering fraction is large, and thus not exhibit the Si feature in absorption, whereas a source with a low covering factor might be seen through an optically thick cloud and show a deep Si feature.

\section{DISCUSSION}

\subsection{Starburst Signatures in Obscured AGNS}

For assigning the ultimate luminosity source of an obscured galaxy, it is necessary to know what fraction arises from an AGN and what fraction from a starburst. Our modeling discussed above indicates that the observed NIR and MIR emissions are mainly produced by dust heated by an AGN. Because of suggestions that the obscuring material may be associated with starbursts within the clouds that surround the AGN, we search for signatures of a starburst component in the spectra of these obscured AGNs. Because of poor $\mathrm{S} / \mathrm{N}$ within the individual spectra (see Fig. 2), there are no confident detections of the features that are diagnostics of a starburst, such as PAHs and low-ionization emission lines. Some of these features might be present in some spectra, but higher $\mathrm{S} / \mathrm{N}$ spectra are required to confirm them. Here we analyze the composite spectra in Figure 8 to search for weak PAH features that are not visible in individual objects.

In none of the composite spectra, however, are there any confident detections of PAH features. Limits can be put on the strength of these features relative to the underlying continuum. 
The spectrum in Figure 8 with the highest limit for a PAH feature is the composite for sources fitted with the T model (Fig. 8a), in which a feature near $7.7 \mu \mathrm{m}$ might be a PAH feature whose peak $F(7.7 \mu \mathrm{m})$ exceeds by $\leq 10 \%$ the flux density of the underlying continuum at $7.7 \mu \mathrm{m}, F(7.7 \mu \mathrm{m})^{\text {cont }}$. If this feature is real, the ratio $F(7.7 \mu \mathrm{m}) / F(7.7 \mu \mathrm{m})^{\text {cont }}$, where $F(7.7 \mu \mathrm{m})$ represents the flux of the total (continuum+line) at $7.7 \mu \mathrm{m}$, would be 1.1. For objects classified as pure starbursts without any AGN contribution, this ratio always exceeds 5 and is typically $\sim 10$ (Genzel et al. 1998; Brandl et al. 2006; Weedman et al. 2006a; Houck et al. 2007). Lower values are observed in some SMGs, but always greater than 2 (Valiante et al. 2007). If a starburst is present in this spectrum, therefore, it is very diluted by the underlying continuum of the AGN component, as seen in other AGNs where PAH emission appears to be weak or absent in the presence of a strong AGN continuum in the MIR (Lutz et al. 1998; Clavel et al. 2000; Valiante et al. 2007).

For use with spectra of poor $\mathrm{S} / \mathrm{N}$ for which the total $\mathrm{PAH}$ fluxes are difficult to determine, Houck et al. (2007) derive an empirical relationship between the luminosity of the $7.7 \mu \mathrm{m}$ feature and the bolometric luminosity of a starburst, $L_{\mathrm{bol}}^{\mathrm{SB}}$, using the starburst spectra and luminosities in Brandl et al. (2006). This relation is

$$
\log L_{\mathrm{bol}}^{\mathrm{SB}}=\log \left[\nu L_{\nu}^{\mathrm{SB}}(7.7 \mu \mathrm{m})\right]+0.78
$$

for $\nu L_{\nu}^{\mathrm{SB}}(7.7 \mu \mathrm{m})$ measured from the $F(7.7 \mu \mathrm{m})$ line at the peak of the $7.7 \mu \mathrm{m}$ feature. If the feature has lower equivalent width than in a pure starburst, as in our composite spectrum, a correction is necessary to subtract the underlying continuum, $F(7.7 \mu \mathrm{m})^{\mathrm{cont}}$, from $F(7.7 \mu \mathrm{m})$ in order to determine $F(7.7 \mu \mathrm{m}){ }^{\text {line }}$ and thus $\nu L_{\nu}^{\mathrm{SB}}(7.7 \mu \mathrm{m})$ that is due to the starburst.

The composite spectrum in Figure $8 a$ also indicates that $\nu F(7.7 \mu \mathrm{m})^{\text {cont }}=\nu F(6 \mu \mathrm{m})$. Therefore, the starburst $7.7 \mu \mathrm{m}$ luminosity can be derived as $\nu L_{\nu}^{\mathrm{SB}}(7.7 \mu \mathrm{m}) \leq 0.1 L_{\text {cont }} \times$ $(7.7 \mu \mathrm{m})=0.1 L(6 \mu \mathrm{m})$. Figure 7 and Table 3 illustrate that the median $\log \left[L(6 \mu \mathrm{m}) /\right.$ ergs s $\left.^{-1}\right]=46.10$, yielding a median $\log \left[\nu L_{\nu}^{\mathrm{SB}}(7.7 \mu \mathrm{m})\right]=45.10$ for the most luminous PAH feature that would satisfy the observed limit in the composite spectrum. This limiting starburst would then have $\log \left(L_{\text {bol }}^{\mathrm{SB}} / \mathrm{ergs} \mathrm{s}^{-1}\right)=$ 45.88 (equal to $\sim 2 \times 10^{12} L_{\odot}$ or to $\mathrm{SFR} \simeq 350 M_{\odot} \mathrm{yr}^{-1}$ ) arising from the starburst, using equation (2). This starburst luminosity, compared to the median AGN bolometric luminosity, $\log \left(L_{\mathrm{bol}}^{\mathrm{AGN}} / \mathrm{ergs} \mathrm{s}^{-1}\right)=46.47$ (see Fig. 7 and Table 3 ), indicates, therefore, that any starburst that is present in the limit of the observed composite spectrum has, on average, a bolometric luminosity $<26 \%$ of the bolometric luminosity arising from the AGN, and thus $<20 \%$ of the total (AGN+starburst) bolometric luminosity.

Eight sources (out of 13 SWIRE sources and five E-FLS sources) are detected in the FIR (70 and/or $160 \mu \mathrm{m})$. For these sources, the FIR luminosities (see Table 3 ) are consistent with those observed in the most luminous starburst galaxies $\left(\sim 10^{13} L_{\odot}\right)$ but exceed the limiting starburst luminosities implied by the absence of PAH features $\left(=10^{45.88} \mathrm{ergs} \mathrm{s}^{-1}=2 \times 10^{12} L_{\odot}\right)$. In two cases (N1_09 and N2_06), these powerful FIR luminosities are consistent with those predicted by the torus models. However, in the other six cases there is evidence for an additional FIR component whose origin is still unclear. The FIR luminosity could arise from a starburst, or from dust far from the torus but still heated by the AGN.

\subsection{Obscuration at High Luminosities}

A comparison between the MIR properties of our sample and other samples from the literature shows that the selected sources can be as luminous as the most luminous MIR-selected type 1
AGNs in the same redshift range. The estimated ratio between obscured and unobscured AGNs at these luminosities $\left(L_{6 \mu \mathrm{m}} \simeq\right.$ $\left.10^{45.5}-10^{46.5} \mathrm{ergs} \mathrm{s}^{-1}\right)$ is about 1.5-1.9, and the surface density is 17-22 obscured QSOs deg ${ }^{-2}$ to the limits of the SWIRE survey.

However, as discussed in the previous sections, the ratio between obscured and unobscured QSOs derived here does not correspond to the ratio between QSOs seen through the obscuring torus and those seen through the torus opening cone. Indeed, from our study, it emerges that the obscuring matter along the LOS is not always associated with the torus. Only about half (12 sources) of obscured QSOs are seen through the torus. Overall and including the unobscured AGN population, we conclude that $35 \%-41 \%$ of MIR-selected red QSOs are unobscured, 37\%-40\% are obscured by the torus, and the remaining $23 \%-25 \%$ are obscured, but not by the torus. These values constrain the covering factor of the torus at these extreme luminosities to be $\sim 37 \%-40 \%$ and the half torus opening angle, measured from the torus axis, to be $\sim 67^{\circ}$. This is significantly larger than the value of $46^{\circ}$ estimated for FIR-selected Seyfert galaxies (Schmitt et al. 2001). Our results thus support the receding torus scenario but are based on the interpretation that a large fraction of obscured QSOs are obscured by dust detached from the torus located along or outside the torus opening cone.

The presence of foreground cold dust detached from the torus that might absorb the nuclear emission was first suggested by Keel (1980) and subsequently supported by other studies at optical, IR, radio, and X-ray wavelengths (Lawrence \& Elvis 1982; Rigby et al. 2006; Ogle et al. 2006; Martínez-Sansigre et al. 2006; Brand et al. 2007; Sajina et al. 2007b; Urrutia et al. 2007). In our Galaxy, a gas-rich spiral, $\tau_{\mathrm{Si}}$ is estimated to be $\sim 2.5$ in an equatorial direction (Rieke et al. 1989). It is possible, therefore, to produce the observed obscuration with dust in a normal spiral galaxy if this host galaxy is viewed edge-on, or in a very disturbed system as a merging galaxy.

Whether the obscuring dust is in a gas-rich spiral host or in a starburst component, it clearly suggests that these extremely luminous and obscured AGNs are not hosted by dust-free elliptical galaxies as commonly seen in luminous AGNs in the nearby universe (e.g., Dunlop et al. 2003). The sources in this study are thus powerful AGNs and might be hosted by dusty galaxies.

The cold absorber will also absorb the nuclear X-ray emission if located along its path. Thus, we can expect that all obscured QSOs, including those obscured only by the cold absorber and those obscured by the torus, will have large column densities in the X-ray $\left(N_{\mathrm{H}}>10^{22} \mathrm{~cm}^{-2}\right)$. Are our predictions consistent with the findings from $\mathrm{X}$-ray studies?

The lack of X-ray data for the majority of the sources in our sample does not allow us to verify that these sources are also absorbed in the X-rays. Only five sources have available X-ray data, and they all show large column densities $\left(N_{\mathrm{H}} \geq 10^{23} \mathrm{~cm}^{-2}\right)$, strongly suggesting that the same might be true for all these sources. At the X-ray luminosities of our sources, $\sim 10^{45} \mathrm{ergs} \mathrm{s}^{-1}$, the observed ratio between X-ray absorbed and unabsorbed AGNs in X-ray-selected AGN samples is, unfortunately, still largely unconstrained, 0.6-1.5:1 (e.g., Akylas et al. 2006; Tajer et al. 2007), mainly because of the difficulty of detecting absorbed sources in the X-rays. These values are lower than our predicted 1.5-1.9:1 ratio. On the other hand, when the selection effects against absorbed sources are minimized and the spectroscopic completeness is maximized, the absorbed-to-unabsorbed fraction can be as high as 2.5:1 (Wang et al. 2007). Since this ratio is higher than our estimates, it is more in agreement with our predictions.

Instead of adding the cold absorber, an alternative way to reproduce the observed SEDs is to include an additional dusty 
component to reproduce the observed NIR emission. The spectrum of this NIR component must decline steeply above $\sim 8 \mu \mathrm{m}$ and thus have a very narrow range of temperatures $>700 \mathrm{~K}$. This NIR component could be produced by direct emission from warm dust around the AGN and not be associated with the torus, e.g., in the NLR (Efstathiou \& Rowan-Robinson 1995; Ruiz et al. 2000; Axon 2001), but models do not usually reproduce the high temperatures observed in our sample (Groves et al. 2006). A more viable origin for this hot dust component might be scattered light in the NIR. This last hypothesis requires the presence of optically thin dust in the NLR that scatters the nuclear NIR emission into the LOS (Efstathiou \& Rowan-Robinson 1995; Pier \& Krolik 1993). This scenario, initially suggested theoretically (Pier \& Krolik 1993; Efstathiou \& Rowan-Robinson 1995), is also supported by speckle interferometric NIR observations of the Seyfert 2 galaxy NGC 1068 (Weigelt et al. 2004). Reconstructed $K$-band images show that the nuclear emission is extended parallel to the outflow and jet direction, instead of perpendicular as expected for the torus. Observations in polarized light would be necessary to test this scenario for our objects. Other possible explanations are a more complex geometry or a different extinction curve.

\section{SUMMARY AND CONCLUSIONS}

In this work, we modeled the IR SEDs and IRS spectra of a sample of $21 \mathrm{MIR}$-selected red AGNs at $z=1.3-3.0$ with extreme MIR luminosities $\left[\nu L_{\nu}(6 \mu \mathrm{m})=L(6 \mu \mathrm{m}) \simeq 10^{46} \mathrm{ergs} \mathrm{s}^{-1}\right]$ with the goal of investigating the properties of their obscuring matter and dependence on luminosity. The sample was drawn from the three largest Spitzer surveys (SWIRE, NDWFS, and FLS) by means of their extremely red infrared colors and presence of a Si absorption feature in their IRS spectra. Eighteen sources clearly show the Si absorption feature with various optical depths (Fig. 2). In the remaining three sources, the $\mathrm{Si}$ absorption is only tentatively observed.

The obscured sources are as MIR luminous as the most luminous MIR-selected type 1 AGNs currently known at similar redshifts, but they are characterized by Si optical depths typical of ULIRGs and Seyfert 2 galaxies, which are usually less luminous MIR sources. The presented sample thus covers a new parameter space in the $L(\mathrm{MIR})-\tau_{\mathrm{Si}}$ region.

The observed SEDs and spectra are fitted with the clumpy torus models developed by Hönig et al. (2006). From the modeling, it emerges that the obscuring matter along the LOS is not always associated with the torus. Only about half ( 12 sources) of obscured QSOs are seen through the torus, and nine sources require, in addition to a torus, a cold absorber to reproduce the depth of the Si feature. The additional component is characterized by large optical depths $\left(\tau_{V}^{\mathrm{CA}}=4-25\right)$. Seven of the sources fitted with the additional cold absorber are characterized by tori at low inclination angles, $\theta=0^{\circ}-30^{\circ}$. The low inclination angles are required by the observed NIR emission, which implies a view of the hottest dust components from the inner parts of the torus. Even though all sources show the Si feature in absorption, we conclude from the modeling that there is no preferred torus inclination in our sample such that these obscured sources have been selected because they are observed through the torus.

The best-fit models indicate that a compact nonflaring torus is preferred by the majority of the sources. The torus emission region is compact (more concentrated toward the nucleus), with the NIR emission being mainly due to dust in the vicinity of the nucleus. The preferred nonflaring torus in the majority of these luminous sources is also consistent with the predictions of the receding torus models (e.g., Simpson 2005; Hönig \& Beckert 2007).
Based on an estimate of the surface density of unobscured QSOs derived from MIR luminosity functions (Brown et al. 2006) and of obscured QSOs derived from a color-selected sample of IR sources, the estimated surface densities are about 17-22 $\mathrm{deg}^{-2}$ for the obscured QSOs and $11.7 \mathrm{deg}^{-2}$ for the unobscured ones at $z=1.3-3.0$. The estimated ratio between obscured and unobscured AGNs at these luminosities $\left[L(6 \mu \mathrm{m}) \simeq 10^{45.5}-\right.$ $\left.10^{46.5} \mathrm{ergs} \mathrm{s}^{-1}\right]$ and redshift range is, thus, $\sim 1.5-1.9$. Overall we find that $\sim 35 \%-41 \%$ of MIR-selected red AGNs are unobscured, $23 \%-25 \%$ are obscured but not by the torus, and the remaining $37 \%-40 \%$ are obscured by the torus. The ratio of obscured to unobscured QSOs constrains the torus half-opening angle, measured from the torus axis, to be $\sim 67^{\circ}$. This value is significantly larger than found for FIR-selected samples of AGNs at lower luminosity $\left(\sim 46^{\circ}\right.$; Schmitt et al. 2001), supporting the receding torus scenario.

Five sources are detected in the X-rays, and large column densities $\left(N_{\mathrm{H}} \geq 10^{23} \mathrm{~cm}^{-2}\right)$ are estimated for all of them. For these objects, the dust covering fraction estimated from the ratio between the MIR and X-ray luminosity is on average higher than what is usually measured in AGNs. However, the large uncertainties and limited subsample of detected X-ray sources do not allow us to confirm such a result. Whether these sources are characterized by an excess of MIR emission with respect to the X-ray emission, and whether this is due to a peculiar dust distribution with respect to the typical AGN population introduced by our selection, is thus not clear.

From the SEDs of the models, we estimate that the AGN bolometric luminosities, $L_{\mathrm{bol}}^{\mathrm{AGN}}$, range from $10^{46}$ to $10^{47} \mathrm{ergs} \mathrm{s}^{-1}$, and the AGN bolometric luminosity can be derived from the $6 \mu \mathrm{m}$ luminosity using the relation $\log L_{\mathrm{bol}}^{\mathrm{AGN}}=\log L(6 \mu \mathrm{m})+0.32 \pm$ 0.06 . The bolometric luminosity of the whole system can be much higher, however, if the luminosity escaping the torus is taken into account and if an additional FIR component is included. Such an FIR component is not included in the AGN torus models, but it is observed in eight cases. In half of the eight FIR-detected sources the torus contribution to the total IR luminosity is lower than that of the additional FIR component. For two of the remaining objects, the two contributions are similar, and in the other two, the torus contribution is larger.

The estimated FIR luminosities for these eight sources are all greater than $3.3 \times 10^{12} L_{\odot}$, which would imply SFRs of $600-$ $3000 M_{\odot} \mathrm{yr}^{-1}$ (Kennicutt 1998) if these FIR luminosities arise from starbursts. However, none of the sources show the PAH features characteristic of starbursts; a limit to the starburst component is estimated from the limit on PAH 7.7 $\mu$ m luminosity in the composite IR spectra. From this limit, we estimate that, on average, the contribution to the bolometric luminosity from a starburst characterized by PAH emission is $\leq 20 \%$ of the total bolometric luminosity. This leaves unanswered the question of whether the extreme FIR luminosities of these sources arise from heavily obscured starbursts or from AGN luminosity heating cool dust that is distant from the torus.

We gratefully acknowledge the anonymous referee for a careful reading of the manuscript and for useful comments and suggestions that improved the paper. M. P. kindly thanks R. Maiolino, N. Levenson, D. J. Axon, and E. Treister for stimulating discussions, B. Siana for providing the optical spectral classification, L. Hao for providing the data used in Figure 13 in machinereadable format, J. Surace for help with the IRAC measurements, M. Lacy for providing information on the latest FLS catalog, S. Croom for his advice on estimating the surface density of 
unobscured type 1 QSOs, and A. Afonso-Luis for providing information on the SWIRE MIPS 70 and $160 \mu$ m observations. M. P. acknowledges financial support from the Marie-Curie Fellowship grant MEIF-CT-2007-042111. This work is based on observations made with the Spitzer Space Telescope, which is operated by the Jet Propulsion Laboratory, California Institute of Technology under NASA contract 1407. Support for this work, part of the Spitzer Space Telescope Legacy Science Program, was provided by NASA through an award issued by the Jet Propulsion Laboratory, California Institute of Technology under NASA contract 1407. Support for this work by the IRS GTO team at Cornell University was provided by NASA through contract 1257184 issued by JPL/Caltech. This work is dedicated to the memory of Harding Eugene (Gene) Smith.

\section{Facilities: Spitzer(IRS,IRAC,MIPS), CXO(ACIS)}

Akylas, A., Georgantopoulos, I., Georgakakis, A., Kitsionas, S., \& Hatziminaoglou, E. 2006, A\&A, 459, 693

Alonso-Herrero, A., et al. 2006, ApJ, 640, 167

Antonucci, R. 1993, ARA\&A, 31, 473

Axon, D. J. 2001, in ASP Conf. Ser. 249, The Central Kiloparsec of Starbursts and AGN: The La Palma Connection, ed. J. H. Knapen et al. (San Francisco: ASP), 193

Barmby, P., et al. 2006, ApJ, 642, 126

Beckert, T., \& Duschl, W. J. 2004, A\&A, 426, 445

Brand, K., et al. 2007, ApJ, 663, 204

Brandl, B. R., et al. 2006, ApJ, 653, 1129

Brown, M. J. I., et al. 2006, ApJ, 638, 88

Chapman, S. C., Smail, I., Blain, A. W., \& Ivison, R. J. 2004, ApJ, 614, 671

Chiar, J. E., et al. 2006, ApJ, 651, 268

Clavel, J., et al. 2000, A\&A, 357, 839

Desai, V., et al. 2006, ApJ, 641, 133

Di Matteo, T., Springel, V., \& Hernquist, L. 2005, Nature, 433, 604

Dullemond, C. P., \& van Bemmel, I. M. 2005, A\&A, 436, 47

Dunlop, J. S., McLure, R. J., Kukula, M. J., Baum, S. A., O’Dea, C. P., \& Hughes, D. H. 2003, MNRAS, 340, 1095

Efstathiou, A., \& Rowan-Robinson, M. 1995, MNRAS, 273, 649

Elvis, M., et al. 1994, ApJS, 95, 1

Fadda, D., et al. 2006, AJ, 131, 2859

Fazio, G. G., et al. 2004, ApJS, 154, 10

Fritz, J., Franceschini, A., \& Hatziminaoglou, E. 2006, MNRAS, 366, 767

Genzel, R., et al. 1998, ApJ, 498, 579

Gliozzi, M., Sambruna, R. M., \& Foschini, L. 2007, ApJ, 662, 878

González-Solares, E. A., et al. 2005, MNRAS, 358, 333

Granato, G. L., Danese, L., \& Franceschini, A. 1997, ApJ, 486, 147

Groves, B., Dopita, M., \& Sutherland, R. 2006, A\&A, 458, 405

Hao, L., Weedman, D. W., Spoon, H. W. W., Marshall, J. A., Levenson, N. A., Elitzur, M., \& Houck, J. R. 2007, ApJ, 655, L77

Hasinger, G., Miyaji, T., \& Schmidt, M. 2005, A\&A, 441, 417

Hatziminaoglou, E., et al. 2005, AJ, 129, 1198

Higdon, S. J. U., et al. 2004, PASP, 116, 975

Ho, L. C. 2005, ApJ, 629, 680

Hönig, S. F., \& Beckert, T. 2007, MNRAS, 380, 1172

Hönig, S. F., Beckert, T., Ohnaka, K., \& Weigelt, G. 2006, A\&A, 452, 459

Hopkins, P. F., Hernquist, L., Cox, T. J., Di Matteo, T., Martini, P., Robertson, B., \& Springel, V. 2005, ApJ, 630, 705

Horst, H., Smette, A., Gandhi, P., \& Duschl, W. J. 2006, A\&A, 457, L17

Houck, J. R., Weedman, D., Le Floc'h, E., Hao, L., \& Soifer, B. T. 2007, ApJ, submitted

Houck, J. R., et al. 2004, ApJS, 154, 18 2005, ApJ, 622, L105

Imanishi, M., Dudley, C. C., Maiolino, R., Maloney, P. R., Nakagawa, T., \& Risaliti, G. 2007, ApJS, 171, 72

Jannuzi, B. T., \& Dey, A. 1999, in ASP Conf. Ser. 191, Photometric Redshifts and the Detection of High Redshift Galaxies, ed. R. Weymann et al. (San Francisco: ASP), 111

Keel, W. C. 1980 , AJ, 85, 198

Kennicutt, R. C., Jr. 1998, ApJ, 498, 541

Krolik, J. H., \& Begelman, M. C. 1988, ApJ, 329, 702

Lacy, M., Petric, A. O., Sajina, A., Canalizo, G., Storrie-Lombardi, L. J., Armus, L., Fadda, D., \& Marleau, F. R. 2007, AJ, 133, 186

Lacy, M., et al. 2004, ApJS, 154, 166

La Franca, F., et al. 2005, ApJ, 635, 864

Lawrence, A. 1991, MNRAS, 252, 586

Lawrence, A., \& Elvis, M. 1982, ApJ, 256, 410

Le Floc'h, E., et al. 2007, ApJ, 660, L65

Levenson, N. A., Heckman, T. M., Krolik, J. H., Weaver, K. A., \& Życki, P. T. 2006, ApJ, 648, 111

Levenson, N. A., Sirocky, M. M., Hao, L., Spoon, H. W. W., Marshall, J. A., Elitzur, M., \& Houck, J. R. 2007, ApJ, 654, L45

Lonsdale, C., et al. 2004, ApJS, 154, 54
Lonsdale, C. J., et al. 2003, PASP, 115, 897

Lutz, D., Maiolino, R., Spoon, H. W. W., \& Moorwood, A. F. M. 2004, A\&A, 418, 465

Lutz, D., Spoon, H. W. W., Rigopoulou, D., Moorwood, A. F. M., \& Genzel, R. 1998, ApJ, 505, L103

Lutz, D., Yan, L., Armus, L., Helou, G., Tacconi, L. J., Genzel, R., \& Baker, A. J. 2005, ApJ, 632, L13

Maiolino, R., Marconi, A., Salvati, M., Risaliti, G., Severgnini, P., Oliva, E., La Franca, F., \& Vanzi, L. 2001, A\&A, 365, 28

Martínez-Sansigre, A., Rawlings, S., Lacy, M., Fadda, D., Jarvis, M. J., Marleau, F. R., Simpson, C., \& Willott, C. J. 2006, MNRAS, 370, 1479

Martínez-Sansigre, A., Rawlings, S., Lacy, M., Fadda, D., Marleau, F. R., Simpson, C., Willott, C. J., \& Jarvis, M. J. 2005, Nature, 436, 666

Murray, S. S., et al. 2005, ApJS, 161, 1

Natta, A., \& Panagia, N. 1984, ApJ, 287, 228

Nenkova, M., Ivezić, Ž., \& Elitzur, M. 2002, ApJ, 570, L9

Ogle, P., Whysong, D., \& Antonucci, R. 2006, ApJ, 647, 161

Osterbrock, D. E., \& Martel, A. 1993, ApJ, 414, 552

Perola, G. C., et al. 2004, A\&A, 421, 491

Pier, E. A., \& Krolik, J. H. 1992, ApJ, 399, L23

‥ 1993, ApJ, 418, 673

Polletta, M., et al. 2006, ApJ, 642, 673

2007, ApJ, 663, 81

Richards, G. T., et al. 2006, ApJS, 166, 470

Rieke, G. H., Rieke, M. J., \& Paul, A. E. 1989, ApJ, 336, 752

Rieke, G. H., et al. 2004, ApJS, 154, 25

Rigby, J. R., Rieke, G. H., Donley, J. L., Alonso-Herrero, A., \& Pérez-González, P. G. 2006, ApJ, 645, 115

Risaliti, G., Elvis, M., Fabbiano, G., Baldi, A., \& Zezas, A. 2005, ApJ, 623, L93

Risaliti, G., Elvis, M., Fabbiano, G., Baldi, A., Zezas, A., \& Salvati, M. 2007, ApJ, 659, L111

Ruiz, M., Alexander, D. M., Young, S., Hough, J., Lumsden, S. L., \& Heisler, C. A. 2000, MNRAS, 316, 49

Sajina, A., Yan, L., Armus, L., Choi, P., Fadda, D., Helou, G., \& Spoon, H. 2007a, ApJ, 664, 713

Sajina, A., Yan, L., Lacy, M., \& Huynh, M. 2007b, ApJ, 667, L17

Sanders, D. B., \& Mirabel, I. F. 1996, ARA\&A, 34, 749

Sanders, D. B., Soifer, B. T., Elias, J. H., Neugebauer, G., \& Matthews, K. 1988, ApJ, 328, L35

Schmitt, H. R., Antonucci, R. R. J., Ulvestad, J. S., Kinney, A. L., Clarke, C. J., \& Pringle, J. E. 2001, ApJ, 555, 663

Shi, Y., et al. 2006, ApJ, 653, 127

Silva, L., Granato, G. L., Bressan, A., \& Danese, L. 1998, ApJ, 509, 103

Simpson, C. 2005, MNRAS, 360, 565

Spergel, D. N., et al. 2003, ApJS, 148, 175

Spoon, H. W. W., Keane, J. V., Tielens, A. G. G. M., Lutz, D., Moorwood, A. F. M., \& Laurent, O. 2002, A\&A, 385, 1022

Spoon, H. W. W., Marshall, J. A., Houck, J. R., Elitzur, M., Hao, L., Armus, L., Brandl, B. R., \& Charmandaris, V. 2007, ApJ, 654, L49

Spoon, H. W. W., et al. 2004, ApJS, 154, 184

Steffen, A. T., Strateva, I., Brandt, W. N., Alexander, D. M., Koekemoer, A. M., Lehmer, B. D., Schneider, D. P., \& Vignali, C. 2006, AJ, 131, 2826

Stern, D., et al. 2005, ApJ, 631, 163

Sturm, E., Hasinger, G., Lehmann, I., Mainieri, V., Genzel, R., Lehnert, M. D., Lutz, D., \& Tacconi, L. J. 2006, ApJ, 642, 81

Sturm, E., Lutz, D., Tran, D., Feuchtgruber, H., Genzel, R., Kunze, D., Moorwood, A. F. M., \& Thornley, M. D. 2000, A\&A, 358, 481

Sturm, E., et al. 2005, ApJ, 629, L21

Surace, J. A., et al. 2005, The SWIRE Data Release 2: Image Atlases and Source Catalogs for ELAIS-N1, ELAIS-N2, XMM-LSS, and the Lockman Hole

Tajer, M., et al. 2007, A\&A, 467, 73

Tozzi, P., et al. 2006, A\&A, 451, 457

Tran, Q. D., et al. 2001, ApJ, 552, 527 
Treister, E., \& Urry, C. M. 2006, ApJ, 652, L79

Ueda, Y., Akiyama, M., Ohta, K., \& Miyaji, T. 2003, ApJ, 598, 886

Urrutia, T., Lacy, M., \& Becker, R. H. 2007, ApJ, in press (arXiv:0709.2805)

Urrutia, T., Lacy, M., Gregg, M. D., \& Becker, R. H. 2005, ApJ, 627, 75

Valiante, E., Lutz, D., Sturm, E., Genzel, R., Tacconi, L. J., Lehnert, M. D., \& Baker, A. J. 2007, ApJ, 660, 1060

Wang, J. X., et al. 2007, ApJ, 657, 95

Weedman, D., et al. 2006a, ApJ, 653, 101

Weedman, D. W., Le Floc'h, E., Higdon, S. J. U., Higdon, J. L., \& Houck, J. R. 2006b, ApJ, 638, 613
Weedman, D. W., et al. 2005, ApJ, 633, 706 2006c, ApJ, 651, 101

Weigelt, G., Wittkowski, M., Balega, Y. Y., Beckert, T., Duschl, W. J., Hofmann, K.-H., Men'shchikov, A. B., \& Schertl, D. 2004, A\&A, 425, 77

Wilkes, B. J., Schmidt, G. D., Cutri, R. M., Ghosh, H., Hines, D. C., Nelson, B., \& Smith, P. S. 2002, ApJ, 564, L65

Yan, L., et al. 2007, ApJ, 658, 778 